\title{
BAERLIN2014 - the influence of land surface types on and the horizontal heterogeneity of air pollutant levels in Berlin
}

Boris Bonn et al.

Correspondence to: Erika von Schneidemesser (evs@iass-potsdam.de)

The copyright of individual parts of the supplement might differ from the CC-BY 3.0 licence. 
The supporting online information document contains additional information on gas- and aerosol properties in different environments. A volatile organic compound (VOC) canister sample in the vicinity of a common air blower in action, which was performed by the Research Centre Juelich, is provided in addition to Table 5, too. The SOI are structured as follows:

S1. Additional information on set-up of instruments, methods applied and comparability of the bicycle measurements with respect to the van

S1.1 Bicycle measurement set-up and calibration factors applied

S1.2 Results of bicycle and van based measurements at comparison tracks

S2. Further information on second flight

S3. Additional gas-phase related results of air pollutants

S3.1 Basic pollutants: $\mathrm{CO}, \mathrm{NO}, \mathrm{NO}_{2}$ and $\mathrm{O}_{3}$

S3.2 Exemplary VOC sample in the vicinity of an air blower in use

S4. Additional information on ambient particulate matter related results

S5. Information about further results - temperature

S6. Comparison of different measurement methods with reference ones in Neukölln

S1. Additional information on set-up of instruments, methods applied and comparability of the bicycle measurements with respect to the van 


\section{S1.1 Bicycle measurement set-up and calibration factors applied}

As stated in section 3.1 of the study, both particle instruments, i.e. the GRIMM1.108 and the DiSCmini, were located in a backpack or a pannier, which sampled ambient air by conductive inlet tubes. These inlet tubes (black silicone for the GRIMM, Tygon for DiSCmini, both ca. 50 $\mathrm{cm}$ in length) and the temperature sensor were fixed on the outside of backpack or pannier (Fig. S1.1). Losses from inlets and tubing were accounted for with correction factors provided in Table A2. 


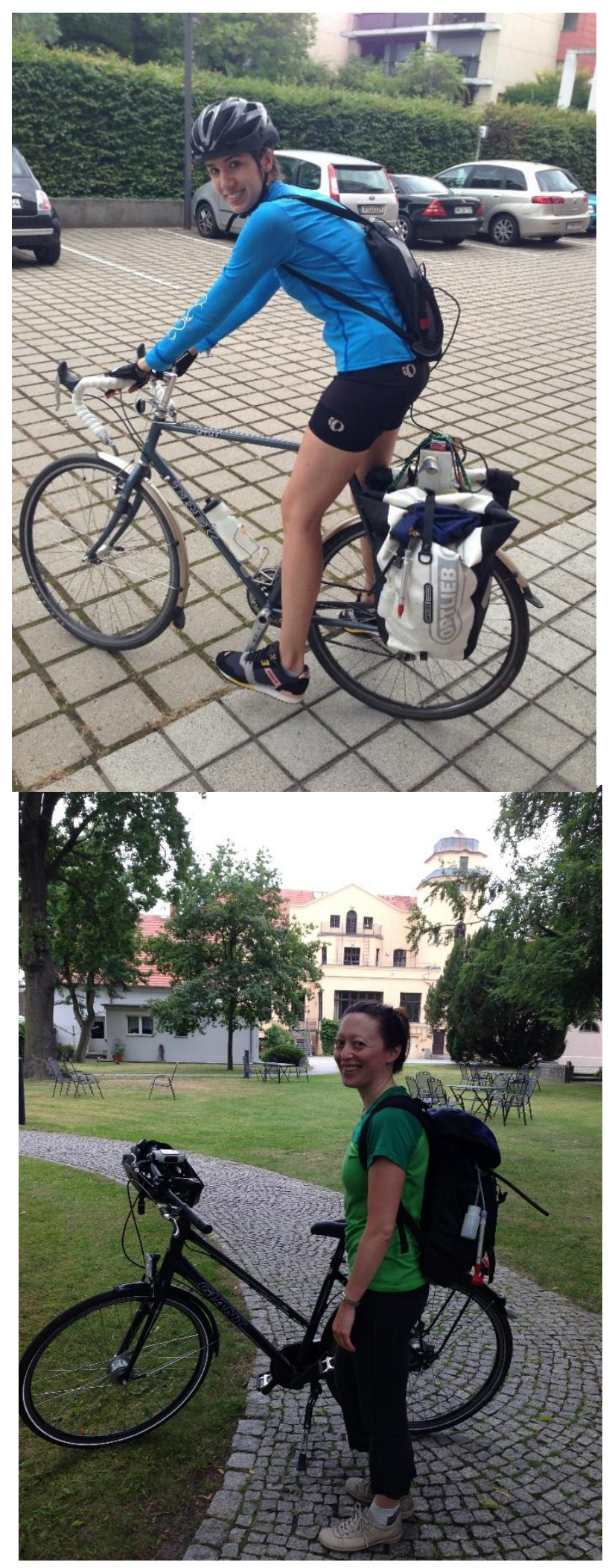


Figure S1.1. Photograph of the measurement set-up used by the cyclists. Instruments were either placed in a pannier at the rear with Tygon inlets about $1 \mathrm{~m}$ above surface (left) or in a backpack of the cyclist with the Tygon inlets at about the same height (saddle).

Based on the particle measurements of the GRIMM instrument its software calculated six particulate mass values corresponding to different size ranges and corresponding to potential health effects: $\mathrm{PM}_{10}, \mathrm{PM}_{2.5}$ and $\mathrm{PM}_{1}$ as well as $\mathrm{PM}$ (inhalable), $\mathrm{PM}$ (thoracic) and PM(alveolar). The final three health-related quantities estimate the particle number concentration for those size fractions making it to the throat/upper respiratory system, lung, and blood system, respectively (EN 481; European Committee for Standardization, 1993).

All particle instruments except the instrument were calibrated a month prior to the campaign in a controlled comparison experiment at TROPOS in Leipzig. Both instruments used on the bicycle measurement platform - DiSCmini and GRIMM 1.108 - were repeatedly operated in parallel with the suite of calibrated particle instruments (GRIMM 1.108, 5.403 and 5.416, and a TSI NSAM provided by the Federal Environmental Agency, Berlin) set up at the reference site in Neukölln. This was used for both instruments to obtain the calibration factors including the inlet losses listed in Table S1.1.

Table S1.1 Correction factors and mean losses for the parameters of both bicycle instruments.

\begin{tabular}{lll}
\hline Instrument and parameter & Correction factor f & Mean loss \\
\hline DiSCmini, tot. part. num. conc. & $1.22 \pm 0.20$ & $18.8 \pm 3.1 \%$ \\
DiSCmini, lung depos. surface area & $1.15 \pm 0.13$ & $13.0 \pm 9.0 \%$ \\
Grimm 1.108, PM10 & $1.24 \pm 0.46$ & $19.3 \pm 7.1 \%$ \\
Grimm 1.108, PM2.5 & $1.24 \pm 0.29$ & $19.6 \pm 4.5 \%$ \\
Grimm 1.108, PM1 & $1.29 \pm 0.12$ & $22.6 \pm 2.1 \%$ \\
Grimm 1.108, PM(inhalable) & $1.28 \pm 0.64$ & $21.7 \pm 10.8 \%$ \\
Grimm 1.108, PM(thoracic) & $1.25 \pm 0.47$ & $19.7 \pm 7.5 \%$
\end{tabular}




\section{S1.2 Results of bicycle and van based measurements at comparison tracks}

Bicycle and van observations were directly compared during two $1.5 \mathrm{~h}$ tracks across Berlin. These focussed on particle number concentration, particulate matter and temperature only, as the bicycle measurements were not equipped for detecting gas-phase pollutants.
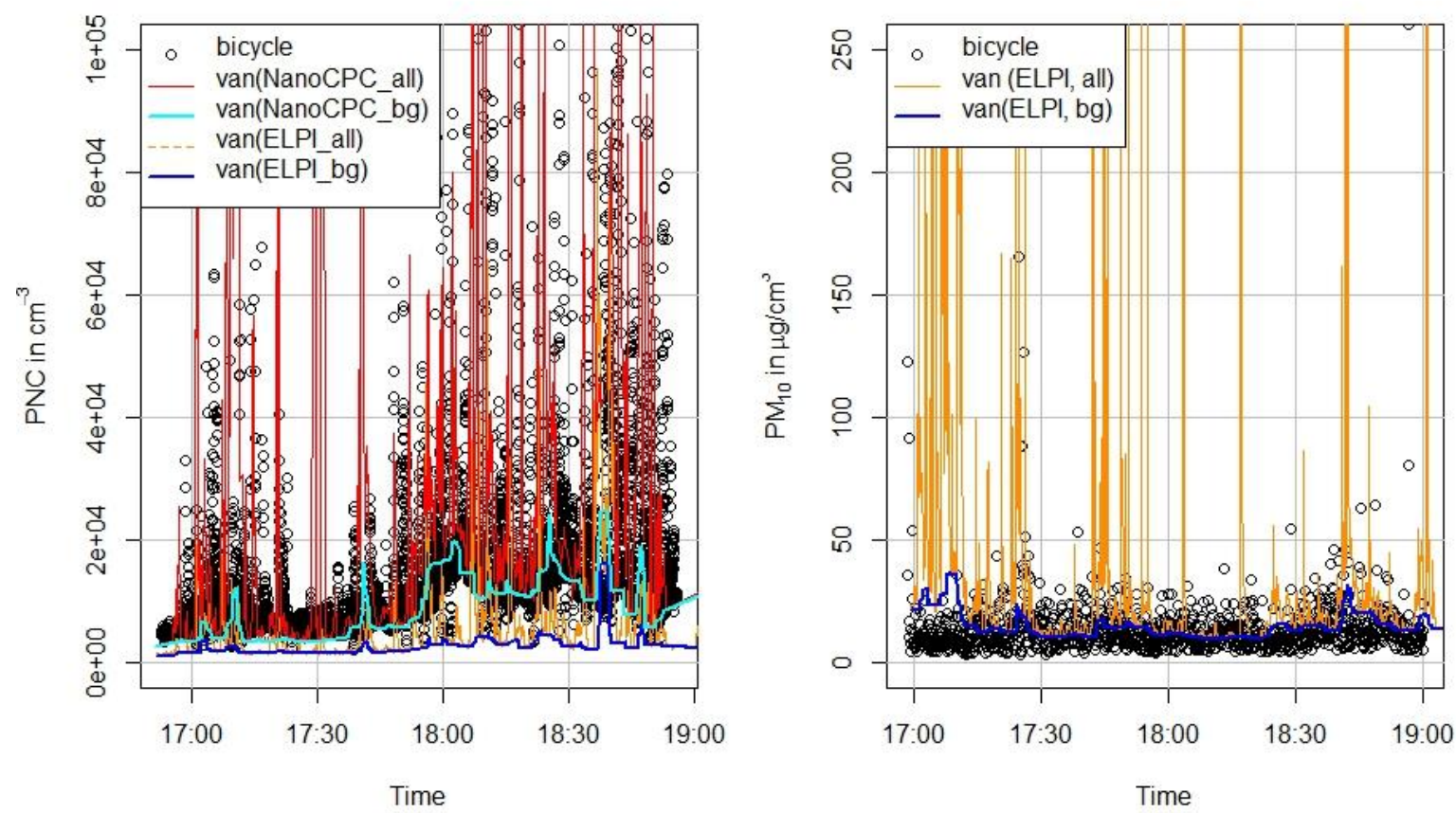


\section{S2. Further information on the second flight}

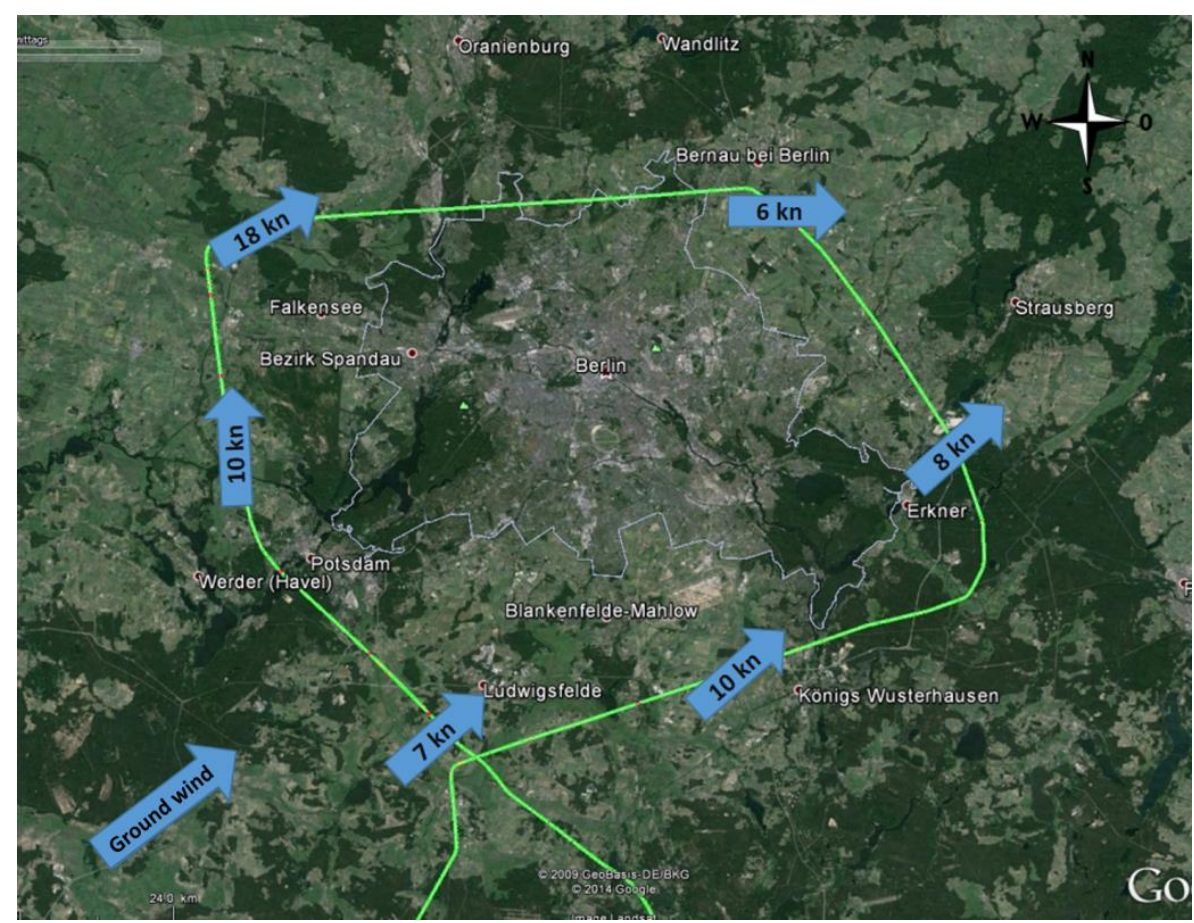

Figure S2.1. Flight track (green line) displayed with wind directions and speeds during the observations at around $500 \mathrm{~m}(1700 \mathrm{ft})$ altitude. 


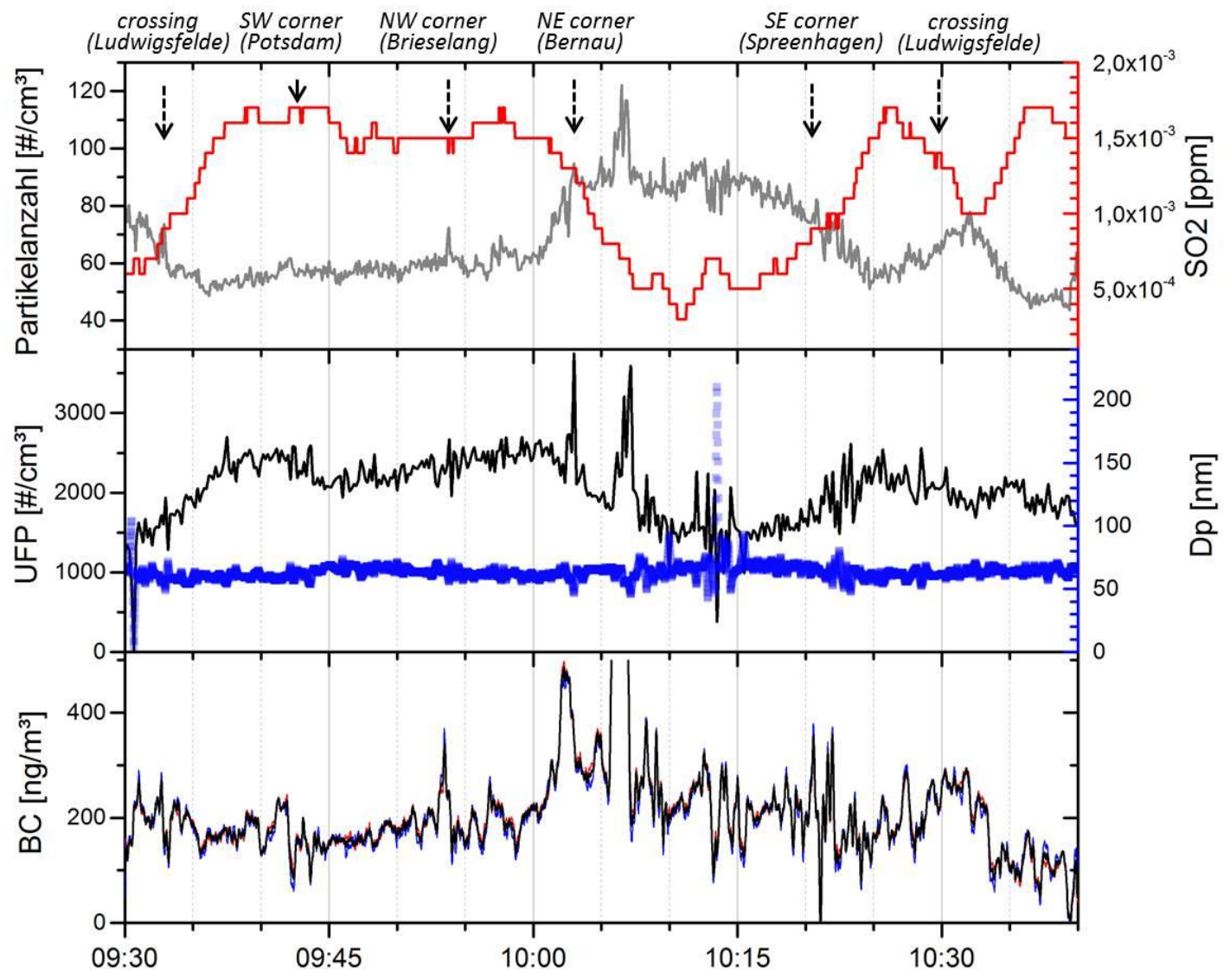

Figure S2.2 Particle parameters measured during the Oct. 10 flight around Berlin. Particle number concentration and sulphur dioxide $\left(\mathrm{SO}_{2}\right)$, UFP and UFP-diameter, Black Carbon (from top position towards bottom) as measured at a constant altitude of around $500 \mathrm{~m}$ (1700 ft).

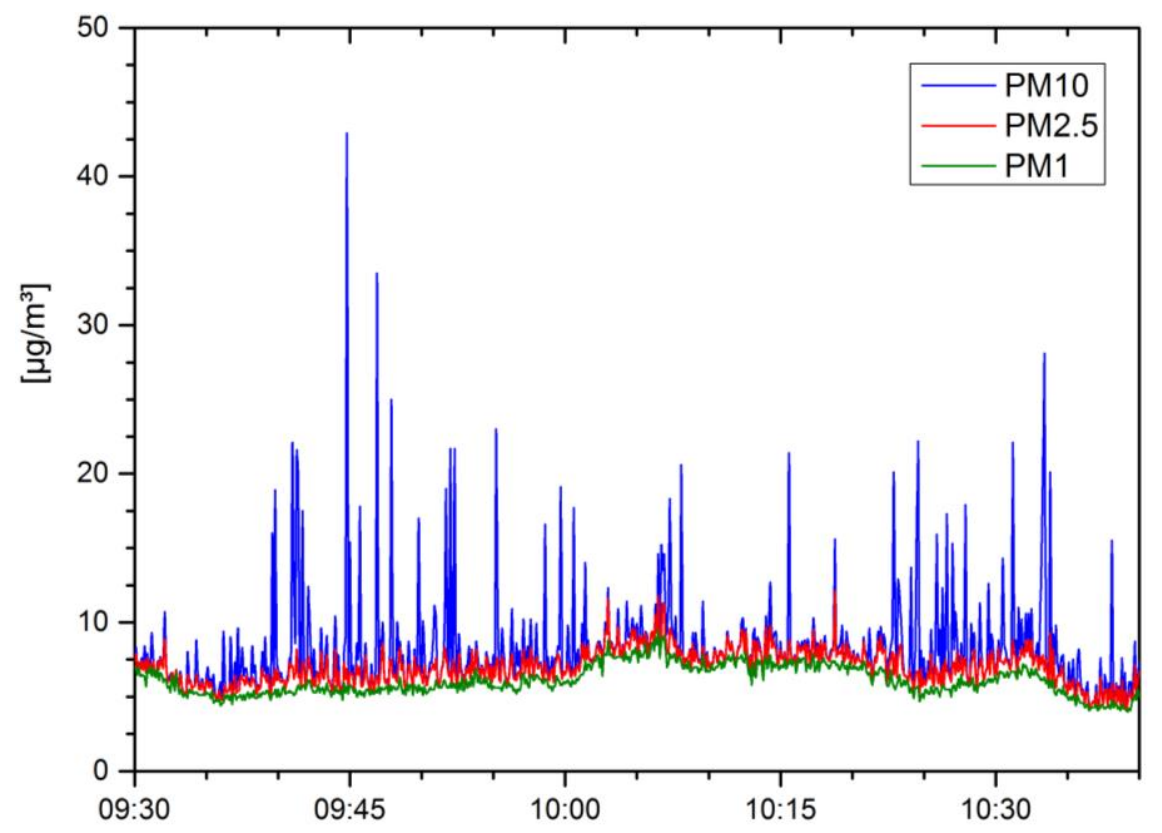


Figure S2.3. Total particle mass (PM) values for different upper cut of diameters provided in $\mu \mathrm{m}$ measured during the flight on the $10^{\text {th }}$ of October 2014. 


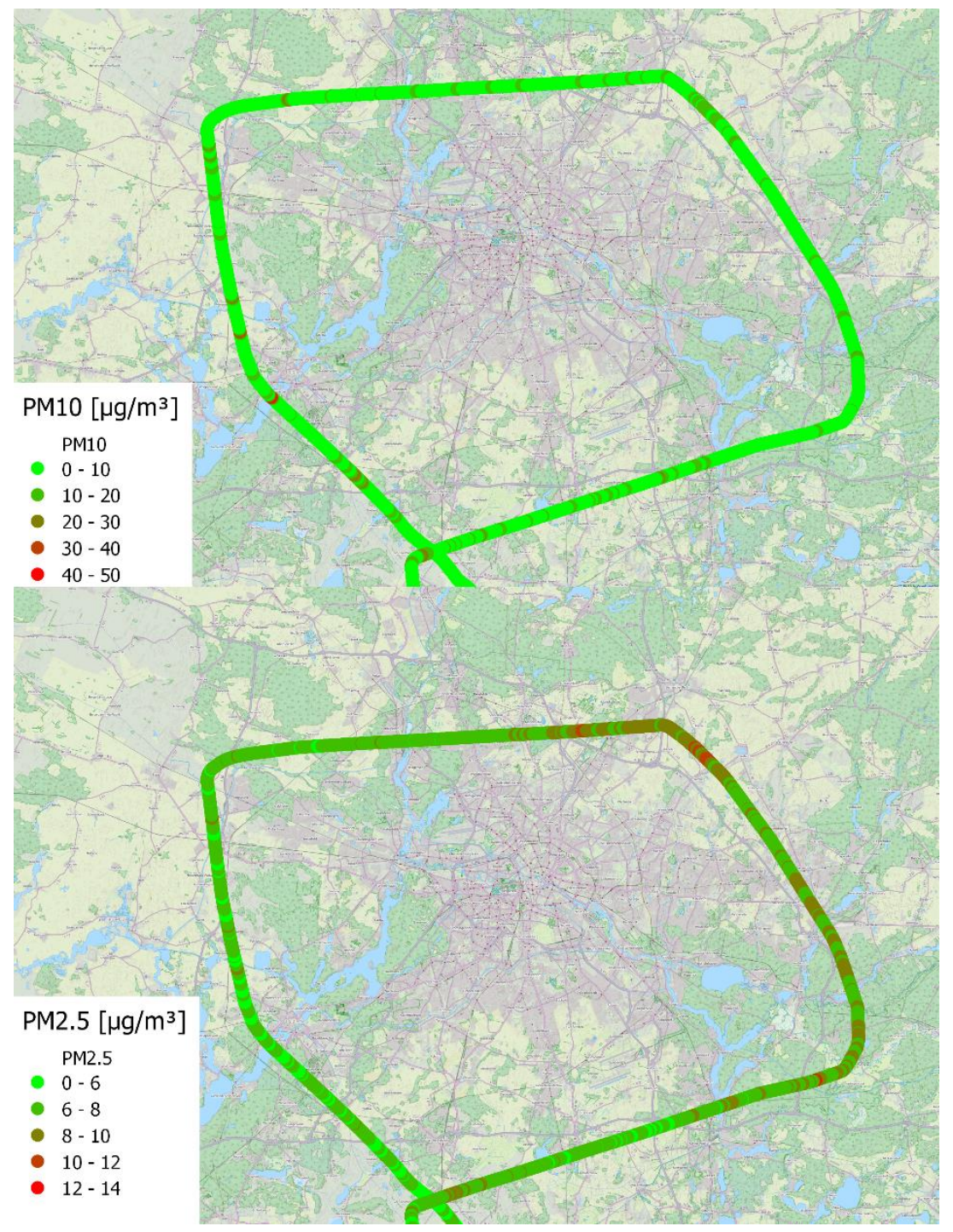

Figure S2.4. Spatial distribution of $\mathrm{PM}_{10}$ (top) and $\mathrm{PM}_{2.5}$ (bottom) flight measurements on the $10^{\text {th }}$ of October 2014. 


\section{S3. Additional gas-phase related results of air pollutants}

\section{S3.1 Basic pollutants: $\mathrm{CO}, \mathrm{NO}, \mathrm{NO}_{2}$ and $\mathrm{O}_{3}$}

Table S3.1.1. Absolute CO characteristics (van measurements, baseline) at different surface usage types. The $25^{\text {th }}, 50^{\text {th }}$ and $75^{\text {th }}$ percentiles, the mean values and the number of data points are provided as columns. “-“ indicates areas, which have not been tested by the method. Values are presented in $\mathrm{ppb}_{\mathrm{v}}$.

\begin{tabular}{|c|c|c|c|c|c|}
\hline surface type & $25 t h$ & median $\left(50^{\text {th }}\right)$ & 75th & mean & no. of data \\
\hline $\begin{array}{l}\text { Reference } \\
\text { (Neukölln): UBA }\end{array}$ & 176 & 201 & 243 & 223.9 & 171237 \\
\hline $\begin{array}{l}\text { Urban block } \\
\text { build. }\end{array}$ & 127.8 & 145.3 & 165.8 & 151.6 & 16501 \\
\hline $\begin{array}{l}\text { Urban single } \\
\text { build. }\end{array}$ & 119.6 & 131.9 & 149.5 & 145.6 & 60257 \\
\hline Industry & 134.1 & 152.7 & 178.9 & 164.8 & 10900 \\
\hline Com.+transp & 149.7 & 163.6 & 183.3 & 173.8 & 4243 \\
\hline Green spaces & 130.0 & 143.7 & 160.6 & 186.4 & 10907 \\
\hline Agriculture & 119.8 & 127.4 & 135.8 & 128.5 & 7877 \\
\hline Dec. forest & 115.1 & 130.0 & 141.6 & 133.6 & 6124 \\
\hline Con. forest & 115.3 & 120.5 & 140.0 & 127.9 & 4508 \\
\hline mix. forest & 121.6 & 131.1 & 142.1 & 133.1 & 1300 \\
\hline
\end{tabular}


Table S3.1.2. Relative CO burden characteristics (van/van (background) measurements) at different surface usage types divided by the reference concentration in Berlin-Neukölln. Listed are the $25^{\text {th }}, 50^{\text {th }}$ and $75^{\text {th }}$ percentiles, the mean values and the number of data points. “_.“ indicates areas, which have not been tested by the method.

\begin{tabular}{llllll} 
surface type & 25 th & median $\left(50^{\text {th }}\right)$ & 75 th & mean & no. of data \\
\hline $\begin{array}{l}\text { Urban } \\
\text { - block build. }\end{array}$ & $0.72 / 0.60$ & $0.90 / 0.67$ & $1.51 / 0.75$ & $11.75 / 0.67$ & 16501 \\
\hline $\begin{array}{l}\text { Urban } \\
\text { single build. }\end{array}$ & $0.64 / 0.57$ & $0.78 / 0.64$ & $1.25 / 0.70$ & $1.46 / 0.65$ & 60257 \\
\hline $\begin{array}{l}\text { Industry } \\
\text { Com.+transp. }\end{array}$ & $0.72 / 0.59$ & $0.93 / 0.66$ & $1.57 / 0.74$ & $1.68 / 0.70$ & 10900 \\
\hline Green spaces & $0.70 / 0.57$ & $1.30 / 0.67$ & $2.34 / 0.75$ & $2.29 / 0.70$ & 4243 \\
\hline Agriculture & $0.63 / 0.59$ & $0.97 / 0.64$ & $2.04 / 0.77$ & $2.08 / 0.80$ & 10907 \\
\hline Dec. forest & $0.56 / 0.52$ & $0.69 / 0.63$ & $0.93 / 0.68$ & $1.11 / 0.64$ & 7877 \\
\hline Con. forest & $0.54 / 0.52$ & $0.70 / 0.57$ & $1.07 / 0.67$ & $1.03 / 0.61$ & 6124 \\
\hline mix. forest & $0.57 / 0.52$ & $0.65 / 0.56$ & $0.88 / 0.63$ & $0.88 / 0.58$ & 4508 \\
\hline
\end{tabular}


Table S3.1.3. Absolute NO characteristics (van measurements) at different surface usage types. The $25^{\text {th }}, 50^{\text {th }}$ and $75^{\text {th }}$ percentiles, the mean values and the number of data points are provided as columns. “_“ indicates areas, which have not been tested by the method. Values are presented in ppb.

\begin{tabular}{|c|c|c|c|c|c|}
\hline surface type & $25 t h$ & median $\left(50^{\text {th }}\right)$ & 75 th & mean & no. of data \\
\hline $\begin{array}{l}\text { Reference } \\
\text { (Neukölln): UBA }\end{array}$ & 0.8 & 1.3 & 2.9 & 3.1 & 11869 \\
\hline $\begin{array}{l}\text { Urban block } \\
\text { build. }\end{array}$ & 6.9 & 32.3 & 106.4 & 86.8 & 1473 \\
\hline $\begin{array}{l}\text { Urban single } \\
\text { build. }\end{array}$ & 4.2 & 22.7 & 88.6 & 81.4 & 5958 \\
\hline Industry & 13.3 & 45.8 & 142.0 & 111.9 & 1021 \\
\hline Com.+transp. & 23.6 & 65.4 & 124.8 & 96.8 & 415 \\
\hline Green spaces & 13.9 & 46.7 & 208.1 & 142.8 & 975 \\
\hline Agriculture & 0.8 & 6.4 & 25.8 & 27.8 & 822 \\
\hline Dec. forest & 1.3 & 10.8 & 38.4 & 30.9 & 654 \\
\hline Con. forest & 1.5 & 5.0 & 28.3 & 28.0 & 561 \\
\hline mix. forest & 2.5 & 11.0 & 46.3 & 43.7 & 136 \\
\hline
\end{tabular}


Table S3.1.4. Relative NO characteristics (van measurements) at different surface usage types divided by the reference concentration in Berlin-Neukölln. Listed are the $25^{\text {th }}, 50^{\text {th }}$ and $75^{\text {th }}$ percentiles, the mean values and the number of data points. “_“ indicates areas, which have not been tested by the method.

\begin{tabular}{lccccc}
$\begin{array}{l}\text { surface type } \\
\text { 25th }\end{array}$ & median $\left(50^{\text {th }}\right)$ & 75 th & mean & no. of \\
\hline $\begin{array}{l}\text { Urban } \\
\text { block build. }\end{array}$ & 2.8 & 15.4 & 60.4 & 74.4 & 1473 \\
\hline $\begin{array}{l}\text { Urban } \\
\text { single build. }\end{array}$ & 2.4 & 16.6 & 73.2 & 80.1 & 5958 \\
\hline $\begin{array}{l}\text { Industry } \\
\text { Com.+transp. }\end{array}$ & 12.9 & 33.9 & 84.5 & & \\
\hline Green spaces & 7.9 & 31.7 & 112.7 & 76.3 & 1021 \\
\hline Agriculture & 0.8 & 6.2 & 24.3 & 31.5 & 758 \\
\hline Dec. forest & 1.0 & 9.1 & 33.4 & 34.4 & 822 \\
\hline Con. forest & 1.5 & 8.0 & 29.9 & 45.8 & 654 \\
\hline mix. forest & 2.4 & 10.5 & 33.5 & 43.8 & 561 \\
\hline
\end{tabular}


Table S3.1.5. Absolute $\mathrm{NO}_{2}$ characteristics (van measurements) at different surface usage types. The $25^{\text {th }}, 50^{\text {th }}$ and $75^{\text {th }}$ percentiles, the mean values and the number of data points are provided as columns. “_“ indicates areas, which have not been tested by the method. Values are presented in ppb.

\begin{tabular}{|c|c|c|c|c|c|}
\hline surface type & 25 th & median $\left(50^{\text {th }}\right)$ & 75th & mean & no. of data \\
\hline $\begin{array}{l}\text { Reference } \\
\text { (Neukölln): UBA }\end{array}$ & 6.7 & 9.4 & 14.1 & 11.8 & 12419 \\
\hline $\begin{array}{l}\text { Urban block } \\
\text { build. }\end{array}$ & 9.2 & 23.9 & 85.3 & 8.8 & 1473 \\
\hline $\begin{array}{l}\text { Urban single } \\
\text { build. }\end{array}$ & 5.8 & 18.1 & 63.2 & 8.1 & 5958 \\
\hline Industry & 12.7 & 30.3 & 97.0 & 8.4 & 1021 \\
\hline Com.+transp. & 20.6 & 56.0 & 148.0 & 5.3 & 352 \\
\hline Green spaces & 9.5 & 29.8 & 108.2 & 7.5 & 758 \\
\hline Agriculture & 1.5 & 5.2 & 23.5 & 3.2 & 732 \\
\hline Dec. forest & 2.6 & 7.6 & 31.0 & 3.3 & 654 \\
\hline Con. forest & 3.4 & 9.3 & 26.0 & 2.9 & 561 \\
\hline mix. forest & 2.2 & 18.0 & 44.1 & 4.7 & 136 \\
\hline
\end{tabular}


Table S3.1.6. Relative $\mathrm{NO}_{2}$ burden characteristics (van measurements) at different surface usage types divided by the reference concentration in Berlin-Neukölln. Listed are the $25^{\text {th }}, 50^{\text {th }}$ and $75^{\text {th }}$ percentiles, the mean values and the number of data points. "-“ indicates areas, which have not been tested by the method.

\begin{tabular}{|c|c|c|c|c|c|}
\hline surface type & 25th & median $\left(50^{\text {th }}\right)$ & 75th & mean & $\begin{array}{c}\text { no. } \\
\text { of data }\end{array}$ \\
\hline $\begin{array}{l}\text { Urban } \\
\text { block build. }\end{array}$ & - 0.6 & 1.6 & 6.3 & 8.8 & 1473 \\
\hline $\begin{array}{l}\text { Urban } \\
\text { single build. }\end{array}$ & - $\quad 0.4$ & 1.3 & 5.1 & 8.1 & 5958 \\
\hline Industry & 0.7 & 2.1 & 6.6 & 8.4 & 1021 \\
\hline Com.ttransp. & 1.0 & 2.6 & 6.6 & 5.3 & 352 \\
\hline green spaces & 0.7 & 1.7 & 7.3 & 7.5 & 758 \\
\hline Agriculture & 0.2 & 0.7 & 2.9 & 3.2 & 822 \\
\hline Dec. forest & 0.2 & 0.5 & 2.5 & 3.3 & 654 \\
\hline Con. forest & 0.2 & 0.7 & 2.1 & 2.9 & 561 \\
\hline mix. forest & 0.2 & 1.6 & 3.4 & 4.7 & 136 \\
\hline
\end{tabular}


Table S3.1.7. Absolute ozone burden characteristics (van measurements) at different surface usage types. The $25^{\text {th }}, 50^{\text {th }}$ and $75^{\text {th }}$ percentiles, the mean values and the number of data points are provided as columns. "_“ indicates areas, which have not been tested by the method. Values are presented in $\mathrm{ppb}$.

\begin{tabular}{|c|c|c|c|c|c|}
\hline surface type & 25 th & median $\left(50^{\text {th }}\right)$ & 75th & mean & no. of data \\
\hline $\begin{array}{l}\text { Reference } \\
\text { (Neukölln): UBA }\end{array}$ & 19.6 & 29.0 & 39.7 & 30.6 & 50158 \\
\hline $\begin{array}{l}\text { Urban block } \\
\text { build. }\end{array}$ & 15.5 & 25.6 & 33.7 & 24.9 & 6549 \\
\hline $\begin{array}{l}\text { Urban single } \\
\text { build. }\end{array}$ & 17.8 & 28.7 & 37.5 & 28.6 & 24503 \\
\hline Industry & 11.7 & 21.7 & 34.1 & 24.0 & 4145 \\
\hline Com.+transp. & 13.2 & 18.9 & 26.4 & 20.6 & 1692 \\
\hline green spaces & 14.7 & 21.6 & 34.6 & 25.1 & 3684 \\
\hline Agriculture & 31.0 & 37.8 & 46.1 & 37.5 & 3267 \\
\hline Dec. forest & 18.3 & 25.7 & 37.5 & 28.0 & 2667 \\
\hline Con. forest & 17.7 & 22.7 & 38.6 & 27.4 & 2099 \\
\hline mix. forest & 17.5 & 27.3 & 43.8 & 30.0 & 548 \\
\hline
\end{tabular}


Table S3.1.8. Relative ozone burden characteristics (van measurements) at different surface usage types divided by the reference concentration in Berlin-Neukölln. Listed are the $25^{\text {th }}, 50^{\text {th }}$ and $75^{\text {th }}$ percentiles, the mean values and the number of data points. "-“" indicates areas, which have not been tested by the method.

\begin{tabular}{|c|c|c|c|c|c|}
\hline surface type & 25 th & median $\left(50^{t h}\right)$ & 75 th & mean & $\begin{array}{l}\text { no. of } \\
\text { data }\end{array}$ \\
\hline $\begin{array}{l}\text { Urban } \\
\text { block build. }\end{array}$ & -0.5 & 0.7 & 0.9 & 0.7 & 6549 \\
\hline $\begin{array}{l}\text { Urban } \\
\text { single build. }\end{array}$ & -0.5 & 0.7 & 0.9 & 0.7 & 23907 \\
\hline Industry & 0.4 & 0.6 & 0.8 & 0.6 & 4145 \\
\hline Com.+transp. & 0.5 & 0.6 & 0.8 & 0.6 & 1692 \\
\hline green spaces & 0.5 & 0.6 & 0.8 & 0.6 & 3684 \\
\hline Agriculture & 0.7 & 0.9 & 0.9 & 0.8 & 3267 \\
\hline Dec. forest & 0.5 & 0.7 & 0.8 & 0.7 & 2667 \\
\hline Con. forest & 0.4 & 0.6 & 0.8 & 0.6 & 2099 \\
\hline mix. forest & 0.5 & 0.7 & 0.9 & 0.7 & 548 \\
\hline
\end{tabular}



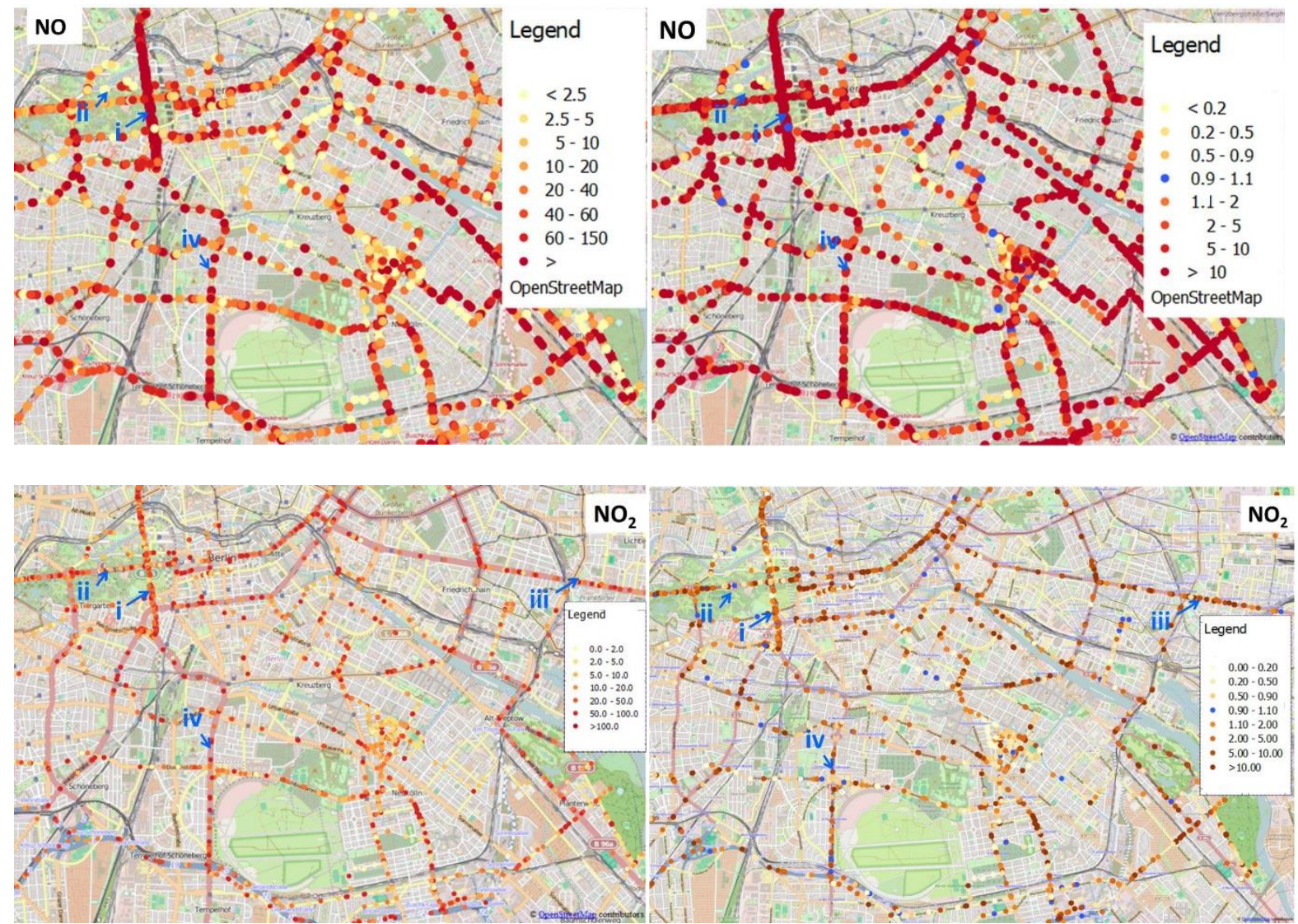

Figure 3.1.1. Mobile measurement data (left) and relative (right) graphs of nitrogen monoxide (NO, top) and dioxide ( $\mathrm{NO}_{2}$, bottom) observed by the measurement van. Mobile measured values are displayed in ppbv. Colours indicate the heterogeneity of the parameters, of the range (mobile measured values) and the variation with respect to reference value. 


\section{S3.2 Exemplary VOC sample in the vicinity of an air blower in use}

Table S3.2.1 Canister sample analysed for VOC compositions in the vicinity of an air blower in use adjoined with the results for the reference site at Nansenstraße. An ozone scrubber was applied in front of the inlet to prevent sampling losses and artefacts. All values are provided as mean volume mixing ratios in ppt $_{\mathrm{v}}$. Elevated anthropogenic compounds with respect to vegetated background area concentration (>average+2STD of the two smaller mixing ratios of vegetated areas) are marked in bold. Underlined numbers mark biogenic compounds exceeding the average of the two smaller mixing ratios for anthropogenic dominated areas +2 standard deviations. "b.d." abbreviates below detection limit.

\begin{tabular}{|c|c|c|}
\hline \multirow[t]{3}{*}{ Compound } & \multicolumn{2}{|c|}{ Locations affect by both emission types } \\
\hline & air blower & Nansenstraße (see Tab. 5) \\
\hline & 1 sample & 14 samples \\
\hline ethene & 33487 & $465 \pm 263$ \\
\hline ethyne & 40003 & $286 \pm 239$ \\
\hline ethane & 5642 & $1686 \pm 1514$ \\
\hline propene & 20445 & $251 \pm 64$ \\
\hline propane & 991 & $825 \pm 613$ \\
\hline propyne & 2574 & $73 \pm 28$ \\
\hline acetaldehyde & 108 & $336 \pm 139$ \\
\hline 2-methylpropane & 1888 & $504 \pm 441$ \\
\hline methanol & 1244 & $4996 \pm 3082$ \\
\hline 1-butene / i-butene & 22974 & $300 \pm 412$ \\
\hline 1,3-butadiene & 1777 & $43 \pm 11$ \\
\hline n-butane & 9867 & b.d. \\
\hline trans-2-butene & b.d. & $16 \pm 3$ \\
\hline cis-2-butene & 1025 & $74 \pm 38$ \\
\hline 1,2-butadiene & 120 & $33 \pm 7$ \\
\hline ethanol & 546 & $333 \pm 189$ \\
\hline 3-methyl-1-butene & b.d. & $52 \pm 6$ \\
\hline 2-methylbutane & 27819 & $465 \pm 178$ \\
\hline acetone & 1002 & $10721 \pm 24004$ \\
\hline
\end{tabular}




\begin{tabular}{|c|c|c|}
\hline 1-pentene & 265 & $35 \pm 8$ \\
\hline 2-propanol & b.d. & $44 \pm 14$ \\
\hline 2-methyl-1-butene & 1544 & b.d. \\
\hline n-pentane & 1883 & $242 \pm 106$ \\
\hline isoprene & $\underline{1012}$ & $266 \pm 159$ \\
\hline trans-2-pentene & 490 & $28 \pm 13$ \\
\hline cis-2-pentene & 230 & $22 \pm 9$ \\
\hline propanal & b.d. & $54 \pm 24$ \\
\hline 2-methyl-2-butene & b.d. & $11 \pm 8$ \\
\hline acetic acid methylic ester & b.d. & b.d. \\
\hline 1,3-pentadiene & b.d. & $14 \pm 4$ \\
\hline cyclopentadiene & b.d. & $35 \pm 14$ \\
\hline 2,2-dimethylbutane & 8847 & $117 \pm 111$ \\
\hline 2-butanol & b.d. & $117 \pm 156$ \\
\hline 1-propanol & b.d. & $342 \pm 377$ \\
\hline cyclopentene & b.d. & $39 \pm 11$ \\
\hline methacrolein & b.d. & $\underline{80 \pm 37}$ \\
\hline \multicolumn{3}{|c|}{ cyclopentane / 2,3-di-methylbutane } \\
\hline & 9838 & $275 \pm 316$ \\
\hline 2-methylpentane & 10049 & $232 \pm 112$ \\
\hline methylvinylketone & b.d. & $\underline{102 \pm}$ \\
\hline butanal & b.d. & $133 \pm 56$ \\
\hline 1-hexene & b.d. & $\underline{113 \pm 68}$ \\
\hline 3-methylpentane & 5651 & $73 \pm 40$ \\
\hline 2-methyl-1-pentene & b.d. & $14 \pm 3$ \\
\hline n-hexane & 373 & $127 \pm 99$ \\
\hline trans-2-hexene & b.d. & $110 \pm 53$ \\
\hline cis-2-hexene & b.d. & $107 \pm 21$ \\
\hline 1,3-hexadiene (trans) & b.d. & $53 \pm 10$ \\
\hline methylcyclopentane & b.d. & $49 \pm 13$ \\
\hline
\end{tabular}




\begin{tabular}{|c|c|c|}
\hline 2,4-dimethylpentane & 8355 & $54 \pm 28$ \\
\hline methylcyclopentene & b.d. & $14 \pm 5$ \\
\hline benzene & 420 & $303 \pm 238$ \\
\hline 1-butanol & b.d. & $28 \pm 14$ \\
\hline cyclohexane & 6705 & $39 \pm 23$ \\
\hline 2-methylhexane & 432 & $36 \pm 14$ \\
\hline 2,3-dimethylpentane & 1169 & $23 \pm 14$ \\
\hline 3-methylhexane & 363 & $82 \pm 34$ \\
\hline pentanal & b.d. & $11 \pm 2$ \\
\hline cyclohexene & b.d. & $18 \pm 4$ \\
\hline 1,3-dimethylcyclo-pentand (cis) & b.d. & $11 \pm 5$ \\
\hline 1-heptene & b.d. & $17 \pm 10$ \\
\hline 2,2,4-trimethyl-pentane & 47404 & $28 \pm 15$ \\
\hline heptane & b.d. & $32 \pm 11$ \\
\hline 2,3-dimethyl-2-pentene & b.d. & b.d. \\
\hline octene & b.d. & b.d. \\
\hline methylcyclohexane & b.d. & $27 \pm 15$ \\
\hline 2,3,4-trimethylpentane & 16235 & $20 \pm 14$ \\
\hline toluene & 16253 & $407 \pm 237$ \\
\hline 2-methylheptane & b.d. & $25 \pm 17$ \\
\hline 4-methylheptane & b.d. & $14 \pm 9$ \\
\hline 3-methylheptane & b.d. & $17 \pm 13$ \\
\hline hexanal & b.d. & $72 \pm 46$ \\
\hline acetic acid butylic ester & b.d. & b.d. \\
\hline n-octane & b.d. & $28 \pm 23$ \\
\hline dimethylcyclo-hexane isomer & b.d. & b.d. \\
\hline ethylbenzene & b.d. & $76 \pm 40$ \\
\hline $\mathrm{m} / \mathrm{p}$-xylene & 235 & $151 \pm 97$ \\
\hline heptanal & b.d. & $22 \pm 14$ \\
\hline
\end{tabular}




\begin{tabular}{|c|c|c|}
\hline styrene & b.d. & $57 \pm 40$ \\
\hline 1-nonene & b.d. & b.d \\
\hline o-xylene & 124 & $64 \pm 38$ \\
\hline n-nonane & b.d. & $21 \pm 4$ \\
\hline i-propylbenzene & b.d. & $30 \pm 70$ \\
\hline$\alpha$-pinene & b.d. & $\underline{31 \pm 26}$ \\
\hline n-propylbenzene & b.d. & $20 \pm 13$ \\
\hline m-ethyltoluene & b.d. & $31 \pm 26$ \\
\hline p-ethyltoluene & b.d. & $24 \pm 14$ \\
\hline 1,3,5-trimethylben-zene (1,3,5-TMB) & b.d. & $46 \pm 55$ \\
\hline sabinene & b.d. & b.d \\
\hline o-ethyltoluene & b.d. & $36 \pm 24$ \\
\hline octanal & b.d. & $13 \pm 5$ \\
\hline$\beta$-pinene & b.d. & $15 \pm 8$ \\
\hline 1,2,4-trimethylbenzene / t-butylbenzene & b.d. & $63 \pm 37$ \\
\hline n-decane & b.d. & $22 \pm 8$ \\
\hline \multirow[t]{2}{*}{ 1,2,3-trimethylbenzene (1,2,3-TMB) } & & \\
\hline & b.d. & $120 \pm 296$ \\
\hline limonene & b.d. & b.d \\
\hline eucalyptol & b.d. & b.d \\
\hline indane & b.d. & b.d \\
\hline 1,3-diethylbenzene & b.d. & $13 \pm 11$ \\
\hline 1,4-diethylbenzene & b.d. & $522 \pm 1380$ \\
\hline butylbenzene & b.d. & b.d.l \\
\hline n-undecane & b.d. & $10 \pm 13$ \\
\hline n-dodecane & b.d. & $26 \pm 24$ \\
\hline n-tridecane & b.d. & b.d \\
\hline
\end{tabular}


Table S4.1. Absolute particle number burden characteristics (bicycle/ van (background) measurements) at different surface usage types. The $25^{\text {th }}, 50^{\text {th }}$ and $75^{\text {th }}$ percentiles, the mean values and the number of data points are provided as columns. "_“" indicates areas, which have not been tested by the method. Values are presented in particles per $\mathrm{cm}^{3}$.

\begin{tabular}{lrrrrr} 
surface type & \multicolumn{1}{l}{ 25th } & median $\left(50^{\text {th }}\right)$ & \multicolumn{1}{l}{ 75th } & mean & no. of data \\
\hline $\begin{array}{l}\text { Reference } \\
\text { (Neukölln): UBA }\end{array}$ & 5930 & 7793 & 10466 & 8697 & 2892 \\
\hline $\begin{array}{l}\text { Urban } \\
\text { block build. }\end{array}$ & $8589 / 7555$ & $13050 / 10114$ & $21155 / 13390$ & $25862 / 11356$ & $55132 / 21646$ \\
\hline Urban & $6021 / 4550$ & $9490 / 6181$ & $15405 / 10078$ & $17044 / 8861$ & $139597 / 81293$ \\
single build. & & & & & \\
\hline Industry & $6269 / 7201$ & $8629 / 10614$ & $16226 / 16710$ & $17330 / 14488$ & $9966 / 13784$ \\
\hline Com.+transp. & $5785 / 9219$ & $8251 / 13780$ & $13101 / 18850$ & $13361 / 17069$ & $4367 / 5637$ \\
\hline green spaces & $4972 / 6441$ & $7563 / 8853$ & $11684 / 16500$ & $13212 / 14828$ & $18913 / 13644$ \\
\hline Agriculture & $-/ 2967$ & $-/ 4869$ & $-/ 7072$ & $-/ 7200$ & $0 / 10576$ \\
\hline Dec. forest & $3613 / 3846$ & $4991 / 5466$ & $8394 / 9169$ & $8657 / 24740$ & $38485 / 8806$ \\
\hline Con. Forest & $3646 / 3501$ & $5802 / 4993$ & $10619 / 5658$ & $12192 / 14630$ & $28726 / 7020$ \\
\hline mix. forest & $3828 / 3501$ & $6059 / 5093$ & $10523 / 7685$ & $11686 / 11860$ & $7215 / 1810$ \\
\hline
\end{tabular}

Table S4.2. Relative particle number burden characteristics (bicycle/ van (background) measurements) at different surface usage types divided by the reference concentration in Berlin- 
Neukölln. Listed are the $25^{\text {th }}, 50^{\text {th }}$ and $75^{\text {th }}$ percentiles, the mean values and the number of data points. “-“ indicates areas, which have not been tested by the method.

\begin{tabular}{lrrrrr} 
surface type & 25th & median $\left(50^{\text {th }}\right)$ & 75th & mean & no. of data \\
\hline $\begin{array}{l}\text { Urban } \\
\text { block build. }\end{array}$ & $1.05 / 0.97$ & $1.58 / 1.42$ & $2.52 / 2.19$ & $3.23 / 1.71$ & $55132 / 21646$ \\
\hline $\begin{array}{l}\text { Urban } \\
\text { single build. }\end{array}$ & $-0.73 / 0.68$ & $1.09 / 0.97$ & $1.87 / 1.43$ & $2.05 / 1.24$ & $139597 / 81293$ \\
\hline Industry & $0.66 / 0.98$ & $0.98 / 1.51$ & $1.83 / 2.04$ & $1.80 / 1.92$ & $9966 / 13784$ \\
\hline Commerc.+transp. & $0.98 / 1.07$ & $1.39 / 1.47$ & $1.88 / 2.09$ & $1.92 / 1.82$ & $4367 / 5637$ \\
\hline Green spaces & $0.57 / 0.82$ & $0.83 / 1.11$ & $1.34 / 1.85$ & $1.47 / 1.70$ & $0 / 10576$ \\
\hline Agriculture & -10.42 & $-/ 0.80$ & $-/ 1.09$ & $-/ 1.16$ & $38485 / 8806$ \\
\hline Dec. forest & $0.58 / 0.47$ & $0.76 / 0.80$ & $1.18 / 1.21$ & $1.28 / 3.06$ & $55132 / 21646$ \\
\hline Con. forest & $0.55 / 0.40$ & $0.85 / 0.69$ & $1.49 / 0.90$ & $1.83 / 1.89$ & $139597 / 81293$ \\
\hline mix. forest & $0.61 / 0.42$ & $0.91 / 0.77$ & $1.57 / 1.09$ & $1.75 / 1.66$ & $9966 / 13784$ \\
\hline
\end{tabular}

Table S4.3 Absolute particle mass $\left(\mathrm{PM}_{10}\right)$ burden characteristics (bicycle/van background (van all) meas.) at different surface usage types provided in $\mu \mathrm{g} / \mathrm{m}^{3}$. Listed are the $25^{\text {th }}, 50^{\text {th }}$ and $75^{\text {th }}$ 
percentiles, the mean values and the number of data points. “-“ indicates areas, which have not been tested by the method.

\begin{tabular}{|c|c|c|c|c|c|}
\hline surface type & $25 t h$ & median $\left(50^{\text {th }}\right)$ & 75 th & mean & no. of data \\
\hline $\begin{array}{l}\text { Reference } \\
\text { (Neukölln): }\end{array}$ & $12.7,11.5$ & $16.6,16.0$ & $22.0,22.4$ & $18.2,18.2$ & 4928,3317 \\
\hline BLUME, UBA & & & & & \\
\hline $\begin{array}{l}\text { Urban } \\
\text { block build. }\end{array}$ & $6.9 / 17.4$ & $13.6 / 32.8$ & $22.7 / 74.7$ & $24.3 / 75.6$ & $8260 / 21801$ \\
\hline $\begin{array}{l}\text { Urban } \\
\text { single build. }\end{array}$ & 7.9/18.6 & $15.0 / 34.3$ & $25.2 / 69.4$ & 29.0/67.9 & $19143 / 82502$ \\
\hline Industry & 13.6/19.6 & $23.9 / 35.9$ & $36.5 / 72.2$ & $30.7 / 73.9$ & $1464 / 14047$ \\
\hline Com.+transp. & $7.4 / 39.5$ & $13.6 / 53.4$ & $23.7 / 77.9$ & $20.1 / 84.1$ & $478 / 5613$ \\
\hline Green spaces & $4.2 / 16.4$ & $9.2 / 31.1$ & $16.1 / 59.7$ & $18.8 / 73.2$ & $2470 / 12976$ \\
\hline Agriculture & $-/ 17.8$ & $-/ 29.5$ & $-/ 46.0$ & $-/ 48.3$ & $0 / 10788$ \\
\hline Dec. forest & $2.8 / 19.1$ & $5.9 / 38.0$ & $10.4 / 71.4$ & $8.9 / 58.2$ & $1842 / 8874$ \\
\hline Con. Forest & $3.2 / 17.8$ & $7.1 / 38.3$ & $12.6 / 70.9$ & $12.7 / 52.7$ & $3410 / 7078$ \\
\hline mix. forest & $3.4 / 15.8$ & $7.8 / 32.7$ & $13.5 / 65.9$ & $13.8 / 53.6$ & $620 / 1820$ \\
\hline
\end{tabular}

Table S4.4. Relative particle mass $\left(\mathrm{PM}_{10}\right)$ burden characteristics (bicycle/van background (van all) meas.) at different surface usage types divided by the corresponding concentrations in Berlin-Neukölln. “_“ indicates areas, which have not been tested by the method. 


\begin{tabular}{lcrrrr} 
surface type & $25 t h$ & median $\left(50^{\text {th }}\right)$ & 75 th & mean & no. of data \\
\hline $\begin{array}{l}\text { Urban } \\
\text { - block build. }\end{array}$ & $0.47 / 0.83(1.14)$ & $0.79 / 1.08(1.75)$ & $1.42 / 1.47(4.75)$ & $1.65 / 1.85(5.53)$ & $7813 / 21801$ \\
\hline $\begin{array}{l}\text { Urban } \\
\text {-single build. }\end{array}$ & $0.47 / 0.91(1.06)$ & $0.78 / 1.11(1.58)$ & $1.36 / 1.54(3.79)$ & $1.89 / 1.58(3.83)$ & $15758 / 82502$ \\
\hline Industry & $0.64 / 0.72(0.94)$ & $1.13 / 1.07(1.56)$ & $2.00 / 1.63(3.87)$ & $1.71 / 1.50(4.15)$ & $1172 / 14047$ \\
\hline Com.+transp. & $0.46 / 1.00(1.40)$ & $0.74 / 1.22(1.83)$ & $1.44 / 1.41(2.79)$ & $1.31 / 1.32(3.34)$ & $478 / 5613$ \\
\hline Green spaces & $0.27 / 0.83(1.02)$ & $0.53 / 1.03(1.42)$ & $0.98 / 1.29(2.85)$ & $1.43 / 1.35(3.92)$ & $2470 / 12976$ \\
\hline Agriculture & $-/ 0.89(0.97)$ & $-/ 1.02(1.20)$ & $-/ 1.24(2.22)$ & $-/ 1.13(2.49)$ & $0 / 10788$ \\
\hline Dec. forest & $0.20 / 0.83(1.07)$ & $0.45 / 1.20(1.94)$ & $0.78 / 2.24(4.20)$ & $0.67 / 1.73(3.57)$ & $1842 / 8874$ \\
\hline Con. forest & $0.23 / 0.84(0.96)$ & $0.53 / 1.10(1.93)$ & $0.89 / 2.67(4.40)$ & $0.87 / 1.83(3.39)$ & $3410 / 7078$ \\
\hline mix. forest & $0.30 / 0.83(0.91)$ & $0.62 / 1.05(1.62)$ & $1.08 / 1.65(3.87)$ & $1.18 / 1.57(3.33)$ & $620 / 1820$ \\
\hline
\end{tabular}

Table S4.5. Absolute particle mass $\left(\mathrm{PM}_{2.5}\right)$ burden characteristics (bicycle/van background (van all) meas.) at different surface usage types provided in $\mu \mathrm{g} / \mathrm{m}^{3}$. Listed are the $25^{\text {th }}, 50^{\text {th }}$ and $75^{\text {th }}$ percentiles, the mean values and the number of data points. “-“ indicates areas, which have not been tested by the method. 


\begin{tabular}{|c|c|c|c|c|c|}
\hline surface type & $25^{\text {th }}$ & median $\left(50^{\text {th }}\right)$ & 75 th & mean & no. of data \\
\hline $\begin{array}{l}\text { Reference (Neu- } \\
\text { kölln): UBA }\end{array}$ & 5.7 & 8.0 & 12.9 & 10.6 & 3317 \\
\hline $\begin{array}{l}\text { Urban } \\
\text { block build. }\end{array}$ & $4.0 / 15.1$ & $6.7 / 26.8$ & $10.0 / 40.6$ & $8.0 / 34.8$ & $8260 / 21801$ \\
\hline $\begin{array}{l}\text { Urban } \\
\text { single build. }\end{array}$ & $4.4 / 15.8$ & $6.8 / 27.2$ & $10.6 / 39.4$ & $8.9 / 35.2$ & $19143 / 82502$ \\
\hline Industry & $6.8 / 19.1$ & $9.8 / 32.1$ & $15.1 / 47.1$ & $12.1 / 39.5$ & $1464 / 14047$ \\
\hline Com.+transp. & $3.9 / 38.2$ & $6.1 / 49.5$ & $8.1 / 66.2$ & $6.6 / 56.2$ & $478 / 5613$ \\
\hline Green spaces & $2.3 / 15.7$ & $4.6 / 27.7$ & $6.9 / 40.5$ & $5.6 / 37.3$ & $2470 / 12976$ \\
\hline Agriculture & $-/ 15.7$ & $-/ 28.2$ & $-/ 33.0$ & $-/ 28.1$ & $0 / 10788$ \\
\hline Dec. Forest & $2.0 / 14.7$ & $3.3 / 24.9$ & $5.2 / 35.2$ & $4.0 / 27.3$ & $1842 / 8874$ \\
\hline Con. Forest & $2.1 / 15.7$ & $3.6 / 23.0$ & $6.0 / 31.1$ & $4.9 / 25.5$ & $3410 / 7078$ \\
\hline mix. forest & $2.5 / 14.2$ & $3.7 / 23.1$ & $5.7 / 34.6$ & $4.6 / 26.8$ & $620 / 1820$ \\
\hline
\end{tabular}

Table S4.6. Relative particle mass $\left(\mathrm{PM}_{2.5}\right)$ burden characteristics (bicycle/van background (van all) meas.) at different surface usage types divided by the corresponding concentrations in Berlin-Neukölln. “_“ indicates areas, which have not been tested by the method. 


\begin{tabular}{lrrrrr} 
surface type & 25 th & median $\left(50^{\text {th }}\right)$ & 75th & mean & no. of data \\
\hline $\begin{array}{l}\text { Urban } \\
\text { - block build. }\end{array}$ & $0.62 / 1.81$ & $0.85 / 2.47$ & $1.15 / 4.41$ & $0.99 / 4.17$ & $7813 / 21801$ \\
\hline $\begin{array}{l}\text { Urban } \\
\text { single build. }\end{array}$ & $0.57 / 1.69$ & $0.81 / 2.26$ & $1.08 / 3.28$ & $1.00 / 3.11$ & $15758 / 82502$ \\
\hline $\begin{array}{l}\text { Industry } \\
\text { Com.+transp. }\end{array}$ & $0.53 / 1.37$ & $1.15 / 2.23$ & $1.57 / 4.16$ & $1.26 / 3.43$ & $1172 / 14047$ \\
\hline Green spaces & $0.36 / 1.71$ & $0.57 / 2.20$ & $0.78 / 3.59$ & $0.68 / 3.30$ & $2470 / 12976$ \\
\hline Agriculture & $-/ 1.32$ & $-/ 1.71$ & $-/ 2.32$ & $-/ 2.13$ & $0 / 10788$ \\
\hline Dec. forest & $0.25 / 1.77$ & $0.43 / 2.41$ & $0.64 / 3.25$ & $0.48 / 2.78$ & $1842 / 8874$ \\
\hline Con. forest & $0.26 / 1.88$ & $0.49 / 2.41$ & $0.72 / 3.49$ & $0.54 / 2.75$ & $3410 / 7078$ \\
\hline mix. forest & $0.34 / 1.31$ & $0.54 / 2.23$ & $0.72 / 3.10$ & $0.60 / 2.69$ & $620 / 1820$ \\
\hline
\end{tabular}

Table S4.7. Absolute particle mass $\left(\mathrm{PM}_{1.0}\right)$ burden characteristics (bicycle/van background (van all) meas.) at different surface usage types provided in $\mu \mathrm{g} / \mathrm{m}^{3}$. Listed are the $25^{\text {th }}, 50^{\text {th }}$ and $75^{\text {th }}$ percentiles, the mean values and the number of data points. “_." indicates areas, which have not been tested by the method. 


\begin{tabular}{|c|c|c|c|c|c|}
\hline surface type & 25 th & median $\left(50^{\text {th }}\right)$ & 75 th & mean & no. of data \\
\hline Reference (Neu- & 3.3 & 5.5 & 10.4 & 8.1 & 3317 \\
\hline \multicolumn{6}{|l|}{ kölln): UBA } \\
\hline Urban & $2.7 / 14.4$ & $4.9 / 23.9$ & $7.0 / 34.3$ & $5.7 / 29.6$ & $8260 / 21801$ \\
\hline \multicolumn{6}{|l|}{ block build. } \\
\hline Urban & $2.8 / 14.8$ & $4.5 / 23.9$ & $6.2 / 33.5$ & $7.2 / 29.5$ & $19143 / 82502$ \\
\hline \multicolumn{6}{|l|}{ single build. } \\
\hline Industry & $4.7 / 18.8$ & $7.2 / 29.1$ & $10.6 / 40.3$ & $9.2 / 34.4$ & $1464 / 14047$ \\
\hline Com.+transp. & $2.4 / 33.8$ & $4.3 / 42.6$ & $5.8 / 55.9$ & $4.4 / 47.9$ & $478 / 5613$ \\
\hline Green spaces & $1.5 / 15.3$ & $3.1 / 25.3$ & $4.8 / 35.8$ & $3.6 / 31.8$ & $2470 / 12976$ \\
\hline Agriculture & $-/ 15.0$ & $-/ 27.4$ & $-/ 30.7$ & $-/ 25.4$ & $0 / 10788$ \\
\hline Dec. forest & $1.5 / 14.1$ & $2.0 / 18.9$ & $2.9 / 28.2$ & $2.6 / 22.3$ & $1842 / 8874$ \\
\hline Con. forest & $1.5 / 14.9$ & $2.1 / 18.1$ & $3.7 / 22.3$ & $3.3 / 20.7$ & $3410 / 7078$ \\
\hline mix. forest & $1.7 / 14.0$ & $2.3 / 18.6$ & $3.4 / 28.4$ & $2.9 / 22.3$ & $620 / 1820$ \\
\hline
\end{tabular}

Table S4.8. Relative particle mass ( $\mathrm{PM}_{1.0}$ ) burden characteristics (bicycle/van background (van all) meas.) at different surface usage types divided by the corresponding concentrations in Berlin-Neukölln. “_“ indicates areas, which have not been tested by the method.

surface type 25 th median $\left(50^{\text {th }}\right) \quad 75^{\text {th }} \quad$ mean no. of data




\begin{tabular}{lrrrrr}
\hline $\begin{array}{l}\text { Urban } \\
\text { block build. }\end{array}$ & $0.68 / 2.49$ & $0.95 / 3.52$ & $1.21 / 5.60$ & $1.04 / 5.11$ & $7813 / 21801$ \\
\hline $\begin{array}{l}\text { Urban } \\
\text { single build. }\end{array}$ & $0.62 / 2.09$ & $0.92 / 2.78$ & $1.14 / 4.23$ & $0.99 / 3.74$ & $15758 / 82502$ \\
\hline Industry & $0.87 / 1.70$ & $1.24 / 2.68$ & $1.60 / 5.23$ & $1.33 / 4.08$ & $1172 / 14047$ \\
\hline Com.+transp. & $0.61 / 2.45$ & $0.83 / 3.05$ & $1.10 / 3.94$ & $0.92 / 3.68$ & $478 / 5613$ \\
\hline Green spaces & $0.39 / 2.28$ & $0.63 / 2.96$ & $0.77 / 4.74$ & $0.63 / 4.06$ & $2470 / 12976$ \\
\hline Agriculture & $-/ 1.53$ & $-/ 1.93$ & $-/ 2.86$ & $-/ 2.52$ & $0 / 10788$ \\
\hline Dec. forest & $0.27 / 2.52$ & $0.47 / 3.05$ & $0.66 / 3.95$ & $0.49 / 3.34$ & $1842 / 8874$ \\
\hline Con. forest & $0.28 / 2.33$ & $0.53 / 3.18$ & $0.73 / 3.88$ & $0.55 / 3.32$ & $3410 / 7078$ \\
\hline mix. forest & $0.35 / 1.51$ & $0.59 / 2.89$ & $0.73 / 3.90$ & $0.58 / 3.21$ & $620 / 1820$ \\
\hline
\end{tabular}

Table S4.9. Absolute particle mass ( $\mathrm{PM}_{\text {inhalable }}$ ) burden characteristics (bicycle/van background (van all) meas.) at different surface usage types provided in $\mu \mathrm{g} / \mathrm{m}^{3}$. Listed are the $25^{\text {th }}, 50^{\text {th }}$ and $75^{\text {th }}$ percentiles, the mean values and the number of data points. “_." indicates areas, which have not been tested by the method.

surface type 25th median $\left(50^{\text {th }}\right) \quad 75$ th mean no. of data




\begin{tabular}{lrrrrr}
\hline $\begin{array}{l}\text { Reference (Neu- } \\
\text { kölln): UBA }\end{array}$ & 13.5 & 18.5 & 26.1 & 20.8 & 3317 \\
\hline $\begin{array}{l}\text { Urban } \\
\text { block build. }\end{array}$ & & & & & \\
\hline Urban & - & & & & \\
single build. & $8.6 / 17.0$ & $14.9 / 31.9$ & $28.0 / 69.2$ & $53.9 / 68.2$ & $8260 / 21801$ \\
\hline Industry & $14.7 / 19.3$ & $28.5 / 35.1$ & $52.8 / 68.2$ & $53.8 / 35.1$ & $1464 / 14047$ \\
\hline Com.+transp. & $7.8 / 38.7$ & $15.0 / 52.1$ & $30.5 / 75.4$ & $36.5 / 78.6$ & $478 / 5613$ \\
\hline Green spaces & $4.4 / 16.1$ & $9.9 / 30.3$ & $18.4 / 56.6$ & $35.5 / 66.6$ & $2470 / 12976$ \\
\hline Agriculture & $-/ 17.4$ & $-/ 28.9$ & -143.8 & $-/ 44.4$ & $0 / 10788$ \\
\hline Dec. forest & $2.9 / 18.4$ & $6.2 / 36.2$ & $11.5 / 64.6$ & $12.3 / 52.6$ & $1842 / 8874$ \\
\hline Con. forest & $3.3 / 17.4$ & $7.5 / 35.6$ & $14.0 / 64.0$ & $20.3 / 47.8$ & $3410 / 7078$ \\
\hline mix. forest & $3.5 / 15.5$ & $8.2 / 31.3$ & $15.2 / 60.6$ & $19.7 / 48.7$ & $620 / 1820$ \\
\hline
\end{tabular}

Table S4.10. Relative particle mass ( $\mathrm{PM}_{\text {inhal }}$ ) burden characteristics (bicycle/van background (van all) meas.) at different surface usage types divided by the corresponding concentrations in Berlin-Neukölln. “_“ indicates areas, which have not been tested by the method.

\begin{tabular}{lrrrrr} 
surface type & $25^{\text {th }}$ & median $\left(50^{\text {th }}\right)$ & 75 th & mean & no. of data \\
\hline $\begin{array}{l}\text { Urban } \\
\text { block build. }\end{array}$ & $0.43 / 0.98$ & $0.75 / 1.51$ & $1.51 / 3.83$ & $3.44 / 4.34$ & $7813 / 21801$ \\
\hline
\end{tabular}




\begin{tabular}{lccccc}
\hline $\begin{array}{l}\text { Urban } \\
\text { single build. }\end{array}$ & $0.42 / 0.93$ & $0.75 / 1.36$ & $1.48 / 3.05$ & $3.68 / 3.07$ & $15758 / 82502$ \\
\hline Industry & $0.58 / 0.82$ & $1.18 / 1.38$ & $2.59 / 3.28$ & $2.73 / 3.36$ & $1172 / 14047$ \\
\hline Com.+transp. & $0.41 / 1.25$ & $0.67 / 1.63$ & $1.54 / 2.45$ & $2.10 / 2.80$ & $478 / 5613$ \\
\hline Green spaces & $0.24 / 0.87$ & $0.49 / 1.24$ & $0.99 / 2.39$ & $2.46 / 3.14$ & $2470 / 12976$ \\
\hline Agriculture & $-/ 0.87$ & $-/ 1.09$ & $-/ 1.92$ & $-/ 2.06$ & $0 / 10788$ \\
\hline Dec. forest & $0.14 / 0.90$ & $0.33 / 1.59$ & $0.59 / 3.29$ & $0.65 / 2.83$ & $1842 / 8874$ \\
\hline Con. forest & $0.16 / 0.82$ & $0.39 / 1.58$ & $0.68 / 3.42$ & $1.01 / 2.68$ & $3410 / 7078$ \\
\hline mix. forest & $0.21 / 0.76$ & $0.46 / 1.37$ & $0.84 / 3.08$ & $1.18 / 2.66$ & $620 / 1820$ \\
\hline
\end{tabular}

Table S4.11. Absolute particle mass ( $\mathrm{PM}_{\text {thoracic }}$ ) burden characteristics (bicycle/van background (van all) meas.) at different surface usage types provided in $\mu \mathrm{g} / \mathrm{m}^{3}$. Listed are the $25^{\text {th }}, 50^{\text {th }}$ and $75^{\text {th }}$ percentiles, the mean values and the number of data points. “_" indicates areas, which have not been tested by the method.

\begin{tabular}{lccccr} 
surface type & 25 th & median $\left(50^{\text {th }}\right)$ & 75th & mean & no. of data \\
\hline $\begin{array}{l}\text { Reference (Neu- } \\
\text { kölln): UBA }\end{array}$ & 12.3 & 17.0 & 23.6 & 19.2 & 3317 \\
\end{tabular}




\begin{tabular}{lcrrrr}
\hline $\begin{array}{l}\text { Urban } \\
\text { block build. }\end{array}$ & $7.2 / 16.9$ & $14.3 / 31.7$ & $24.7 / 66.8$ & $27.4 / 64.0$ & $8260 / 21801$ \\
\hline $\begin{array}{l}\text { Urban } \\
\text { single build. }\end{array}$ & $8.2 / 18.1$ & $16.1 / 32.6$ & $27.2 / 60.7$ & $32.7 / 58.8$ & $19143 / 82502$ \\
\hline Industry & $14.2 / 19.3$ & $25.9 / 34.8$ & $39.8 / 66.3$ & $33.8 / 64.0$ & $1464 / 14047$ \\
\hline Com.+transp. & $7.7 / 38.6$ & $14.4 / 52.0$ & $26.2 / 74.8$ & $26.0 / 76.0$ & $478 / 5613$ \\
\hline Green spaces & $4.4 / 16.1$ & $9.7 / 30.1$ & $17.3 / 55.2$ & $20.9 / 63.1$ & $2470 / 12976$ \\
\hline Agriculture & $-/ 17.3$ & $-/ 28.8$ & $-/ 42.7$ & $-/ 42.2$ & $0 / 10788$ \\
\hline Dec. forest & $2.9 / 18.2$ & $6.2 / 35.5$ & $11.1 / 61.7$ & $9.5 / 49.7$ & $1842 / 8874$ \\
\hline Con. Forest & $3.3 / 17.3$ & $7.5 / 34.6$ & $13.4 / 61.2$ & $13.8 / 45.3$ & $3410 / 7078$ \\
\hline mix. forest & $3.5 / 15.5$ & $8.1 / 30.7$ & $14.5 / 57.8$ & $15.1 / 46.1$ & $620 / 1820$ \\
\hline
\end{tabular}

Table S4.12. Relative particle mass $\left(\mathrm{PM}_{\text {thor }}\right)$ burden characteristics (bicycle/van background (van all) meas.) at different surface usage types divided by the corresponding concentrations in Berlin-Neukölln. “-“ indicates areas, which have not been tested by the method.

\begin{tabular}{lrrrrr} 
surface type & $25^{\text {th }}$ & median $\left(50^{\text {th }}\right)$ & $75^{\text {th }}$ & mean & no. of data \\
\hline $\begin{array}{l}\text { Urban } \\
\text { block build. }\end{array}$ & $0.46 / 1.06$ & $0.78 / 1.60$ & $1.46 / 3.88$ & $1.77 / 4.39$ & $7813 / 21801$ \\
\hline $\begin{array}{l}\text { Urban } \\
\text { single build. }\end{array}$ & $0.46 / 0.99$ & $0.78 / 1.45$ & $1.39 / 3.13$ & $2.02 / 3.11$ & $15758 / 82502$ \\
\hline
\end{tabular}




\begin{tabular}{lrrrrr}
\hline Industry & $0.63 / 0.88$ & $1.15 / 1.46$ & $2.06 / 3.40$ & $1.78 / 3.39$ & $1172 / 14047$ \\
\hline Com.+transp. & $0.44 / 1.32$ & $0.74 / 1.71$ & $1.47 / 2.56$ & $1.36 / 2.96$ & $478 / 5613$ \\
\hline Green spaces & $0.26 / 0.95$ & $0.53 / 1.32$ & $1.00 / 2.49$ & $1.51 / 3.38$ & $2470 / 12976$ \\
\hline Agriculture & $-/ 0.92$ & $-/ 1.13$ & $-/ 1.94$ & $-/ 2.18$ & $0 / 10788$ \\
\hline Dec. forest & $0.16 / 0.99$ & $0.36 / 1.70$ & $0.63 / 3.40$ & $0.54 / 2.86$ & $1842 / 8874$ \\
\hline Con. forest & $0.17 / 0.89$ & $0.42 / 1.67$ & $0.72 / 3.51$ & $0.73 / 2.72$ & $3410 / 7078$ \\
\hline mix. forest & $0.23 / 0.83$ & $0.50 / 1.46$ & $0.86 / 3.17$ & $0.98 / 2.68$ & $620 / 1820$ \\
\hline
\end{tabular}

Table S4.13. Absolute particle mass $\left(\mathrm{PM}_{\mathrm{alveolic}}\right)$ burden characteristics (bicycle/van background (van all) meas.) at different surface usage types provided in $\mu \mathrm{g} / \mathrm{m}^{3}$. Listed are the $25^{\text {th }}, 50^{\text {th }}$ and $75^{\text {th }}$ percentiles, the mean values and the number of data points. “_“ indicates areas, which have not been tested by the method.

\begin{tabular}{lccccr} 
surface type & $25^{\text {th }}$ & median $\left(50^{\text {th }}\right)$ & $75^{\text {th }}$ & mean & no. of data \\
\hline $\begin{array}{l}\text { Reference } \\
\text { (Neu-kölln): }\end{array}$ & 8.2 & 11.5 & 16.1 & 13.6 & 3317 \\
UBA & & & & & \\
\hline
\end{tabular}




\begin{tabular}{lccccc}
\hline $\begin{array}{l}\text { Urban } \\
\text { block build. }\end{array}$ & $5.3 / 16.6$ & $9.2 / 31.1$ & $13.9 / 62.1$ & $11.8 / 58.3$ & $8260 / 21801$ \\
\hline $\begin{array}{l}\text { Urban } \\
\text { single build. }\end{array}$ & $6.1 / 17.7$ & $9.9 / 31.9$ & $15.5 / 54.9$ & $13.5 / 53.9$ & $19143 / 82502$ \\
\hline Industry & $9.9 / 19.2$ & $13.5 / 34.6$ & $20.1 / 62.6$ & $16.4 / 59.2$ & $1464 / 14047$ \\
\hline Com.ttransp. & $5.1 / 38.5$ & $8.9 / 51.3$ & $13.0 / 73.0$ & $10.4 / 71.7$ & $478 / 5613$ \\
\hline Green spaces & $3.4 / 16.1$ & $6.6 / 29.9$ & $10.2 / 52.6$ & $9.2 / 58.0$ & $2470 / 12976$ \\
\hline Agriculture & $-/ 17.1$ & $-/ 28.7$ & $-/ 41.2$ & $-/ 39.5$ & $0 / 10788$ \\
\hline Dec. forest & $2.6 / 17.7$ & $4.7 / 34.0$ & $7.4 / 55.5$ & $5.8 / 45.1$ & $1842 / 8874$ \\
\hline Con. Forest & $2.9 / 17.1$ & $5.3 / 32.3$ & $8.7 / 54.4$ & $7.2 / 41.1$ & $3410 / 7078$ \\
\hline mix. forest & $3.2 / 15.4$ & $5.6 / 29.6$ & $8.4 / 53.0$ & $7.4 / 42.2$ & $620 / 1820$ \\
\hline
\end{tabular}

Table S4.14. Relative particle mass ( $\left.\mathrm{PM}_{\mathrm{alveol}}\right)$ burden characteristics (bicycle/van background (van all) meas.) at different surface usage types divided by the corresponding concentrations in Berlin-Neukölln. “-“ indicates areas, which have not been tested by the method.

\begin{tabular}{lrrrrr} 
surface type & $25^{\text {th }}$ & median $\left(50^{\text {th }}\right)$ & $75^{\text {th }}$ & mean & no. of data \\
\hline $\begin{array}{l}\text { Urban } \\
\text { block build. }\end{array}$ & $0.56 / 1.44$ & $0.81 / 2.14$ & $1.17 / 5.04$ & $1.06 / 5.58$ & $7813 / 21801$ \\
\hline $\begin{array}{l}\text { Urban } \\
\text { single build. }\end{array}$ & $0.53 / 1.36$ & $0.79 / 1.98$ & $1.11 / 3.93$ & $1.12 / 3.89$ & $15758 / 82502$ \\
\hline
\end{tabular}




\begin{tabular}{lccccc}
\hline Industry & $0.78 / 1.15$ & $1.06 / 1.92$ & $1.45 / 4.46$ & $1.25 / 4.24$ & $1172 / 14047$ \\
\hline Com.+transp. & $0.53 / 1.73$ & $0.73 / 2.23$ & $1.14 / 3.21$ & $1.01 / 3.53$ & $341 / 4875$ \\
\hline Green spaces & $0.34 / 1.31$ & $0.57 / 1.80$ & $0.82 / 3.33$ & $0.87 / 4.05$ & $1961 / 9598$ \\
\hline Agriculture & $-/ 1.14$ & $-/ 1.41$ & $-/ 2.37$ & $-/ 2.53$ & $0 / 9488$ \\
\hline Dec. forest & $0.21 / 1.38$ & $0.40 / 2.34$ & $0.63 / 4.26$ & $0.48 / 3.61$ & $1842 / 8874$ \\
\hline Con. forest & $0.24 / 1.30$ & $0.48 / 2.31$ & $0.73 / 4.39$ & $0.56 / 3.48$ & $3410 / 7078$ \\
\hline mix. forest & $0.30 / 1.22$ & $0.54 / 2.03$ & $0.80 / 4.08$ & $0.68 / 3.37$ & $620 / 1820$ \\
\hline
\end{tabular}

Table S4.15. Absolute lung deposable surface area (LDSA) characteristics (bicycle) at different surface usage types in $\mu \mathrm{m}^{2} / \mathrm{cm}^{3}$. The $25^{\text {th }}, 50^{\text {th }}$ and $75^{\text {th }}$ percentiles, the mean values and the number of data points are provided as columns. “_“" indicates areas, which have not been tested by the method. Values are presented in particles per $\mathrm{cm}^{3}$.

\begin{tabular}{lccccc} 
surface type & $25^{\text {th }}$ & median $\left(50^{\text {th }}\right)$ & $75^{\text {th }}$ & mean & no. of data \\
\hline $\begin{array}{l}\text { Reference } \\
\begin{array}{l}\text { (Neukölln): UBA } \\
\text { (Neurk }\end{array}\end{array}$ & $\mathbf{2 4 . 3}$ & $\mathbf{2 5 . 8}$ & $\mathbf{3 3 . 5}$ & $\mathbf{2 8 . 8}$ & $\mathbf{3 7 6 3 2}$ \\
\hline $\begin{array}{l}\text { Urban } \\
\text { build. }\end{array}$ & & & & & \\
\hline
\end{tabular}




\begin{tabular}{|c|c|c|c|c|c|}
\hline $\begin{array}{l}\text { Urban } \\
\text { build. }\end{array}$ & 16.5 & 24.7 & 38.2 & 34.3 & 139597 \\
\hline Industry & 16.5 & 25.8 & 41.6 & 37.4 & 9966 \\
\hline Commerc.+transport & 12.5 & 15.4 & 21.1 & 21.5 & 5335 \\
\hline Green spaces & 12.7 & 19.1 & 32.3 & 28.2 & 18913 \\
\hline Dec. forest & 9.8 & 15.0 & 22.1 & 20.6 & 38485 \\
\hline Con. forest & 12.6 & 17.5 & 27.8 & 24.3 & 28726 \\
\hline mix. forest & 11.8 & 15.6 & 23.7 & 22.4 & 7215 \\
\hline
\end{tabular}

Table S4.16. Relative LDSA characteristics (bicycle) at different surface usage types divided by the reference concentration in Berlin-Neukölln. Listed are the $25^{\text {th }}, 50^{\text {th }}$ and $75^{\text {th }}$ percentiles, the mean values and the number of data points. “_“ indicates areas, which have not been tested by the method.

\begin{tabular}{lccccc} 
surface type & $25^{\text {th }}$ & median $\left(50^{\text {th }}\right)$ & $75^{\text {th }}$ & mean & no. of data \\
\hline $\begin{array}{l}\text { Urban } \\
\text { - block build. }\end{array}$ & 0.86 & 1.15 & 1.76 & 1.77 & 55132 \\
\hline $\begin{array}{l}\text { Urban } \\
\text {-single build. }\end{array}$ & 0.65 & 0.92 & 1.44 & 1.32 & 139597 \\
\hline
\end{tabular}




\begin{tabular}{llllll}
\hline Industry & 0.73 & 1.13 & 1.72 & 1.52 & 9966 \\
\hline Com.+transp. & 0.61 & 0.71 & 0.89 & 0.91 & 5335 \\
\hline green spaces & 0.50 & 0.71 & 1.01 & 0.97 & 18913 \\
\hline Dec. forest & 0.49 & 0.63 & 0.84 & 0.81 & 38485 \\
\hline Con. forest & 0.49 & 0.68 & 1.07 & 0.96 & 28726 \\
\hline mix. forest & 0.51 & 0.67 & 0.98 & 0.94 & 7215 \\
\hline
\end{tabular}

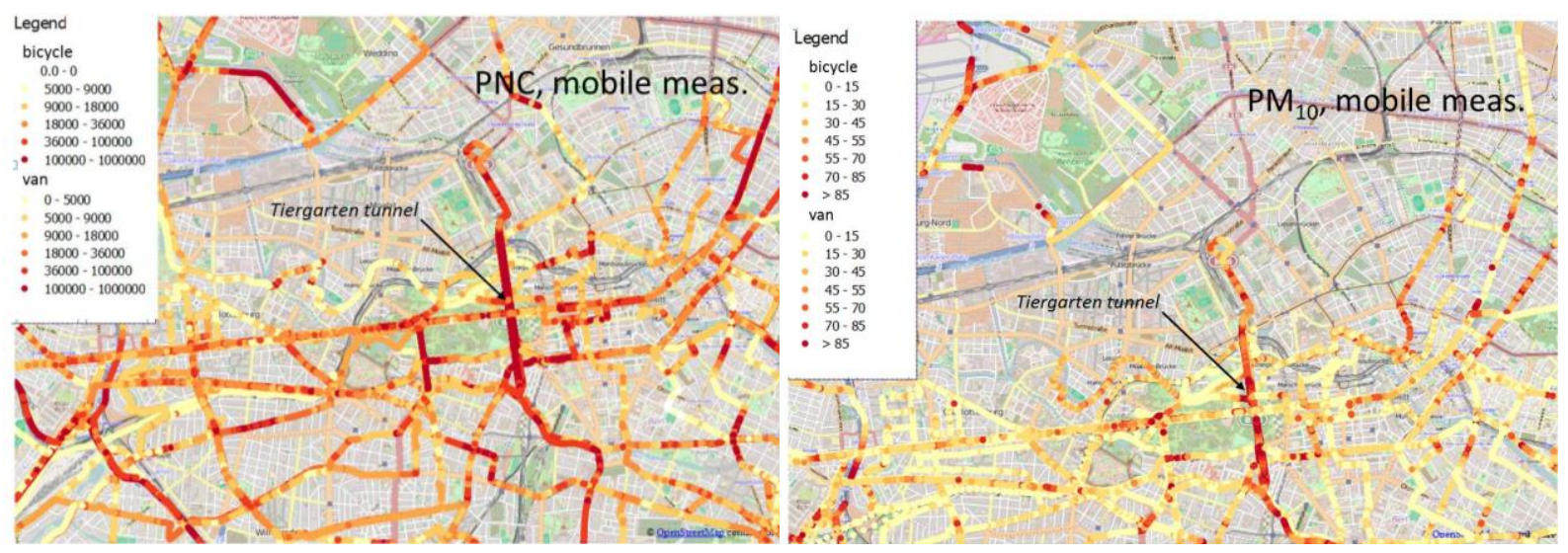

Figure S4.1 Zoomed heterogeneity of particle number (left) and mass ( $\mathrm{PM}_{10}$, right) concentrations in the center of Berlin displayed in absolute measured values. This figure is an extension of Fig. 8. 


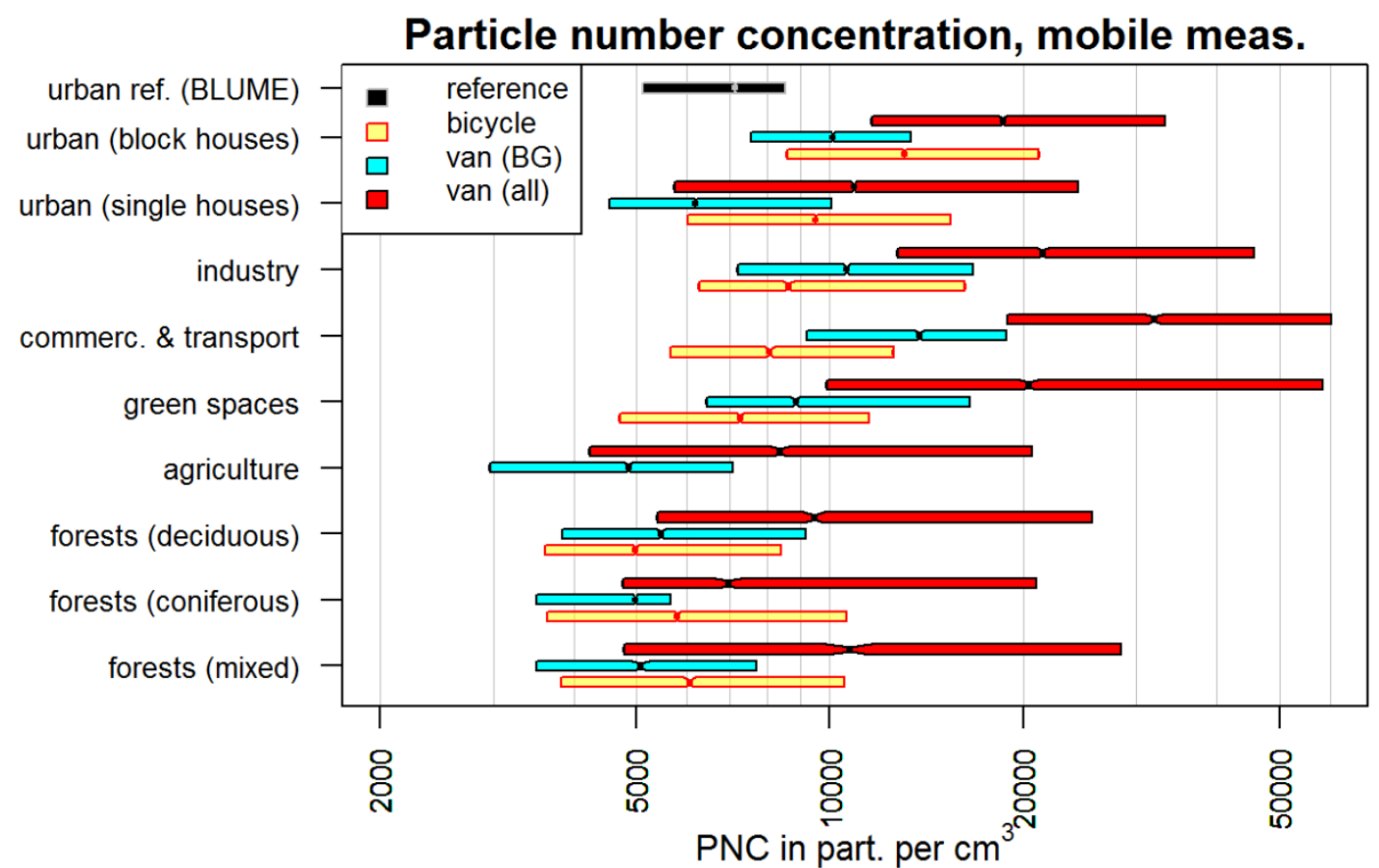

Particle number concentration, relative

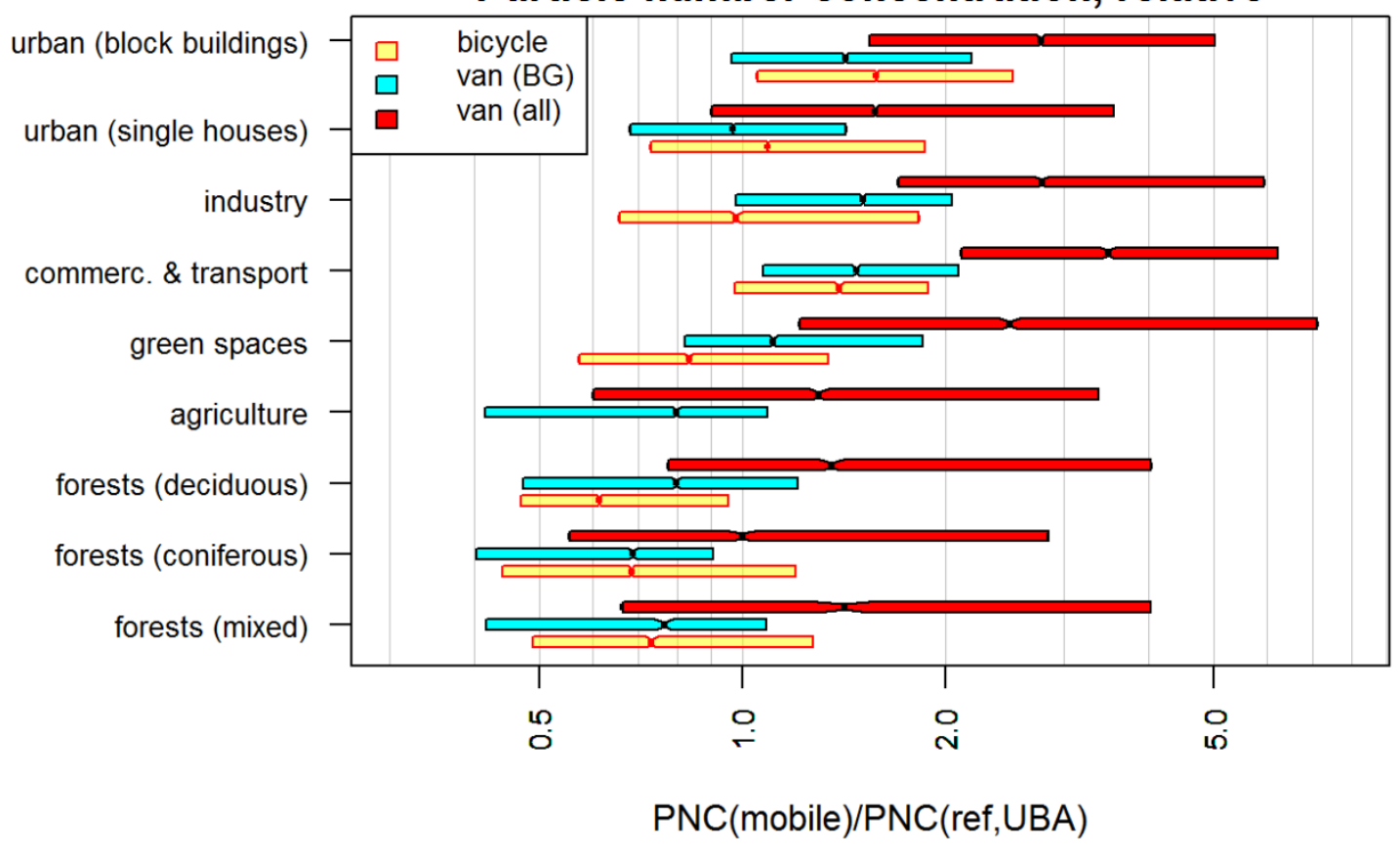

Figure S4.2 Boxplots of particle number concentration ratios for different land surface types (CORINE) and different observation platforms compared to the measurements in BerlinNeukölln. The boxplots range from the $25^{\text {th }}$ to the $75^{\text {th }}$ percentile with notches from the $45^{\text {th }}$ to the $55^{\text {th }}$ percentile centered on the median. 
S5. Information about further results - temperature

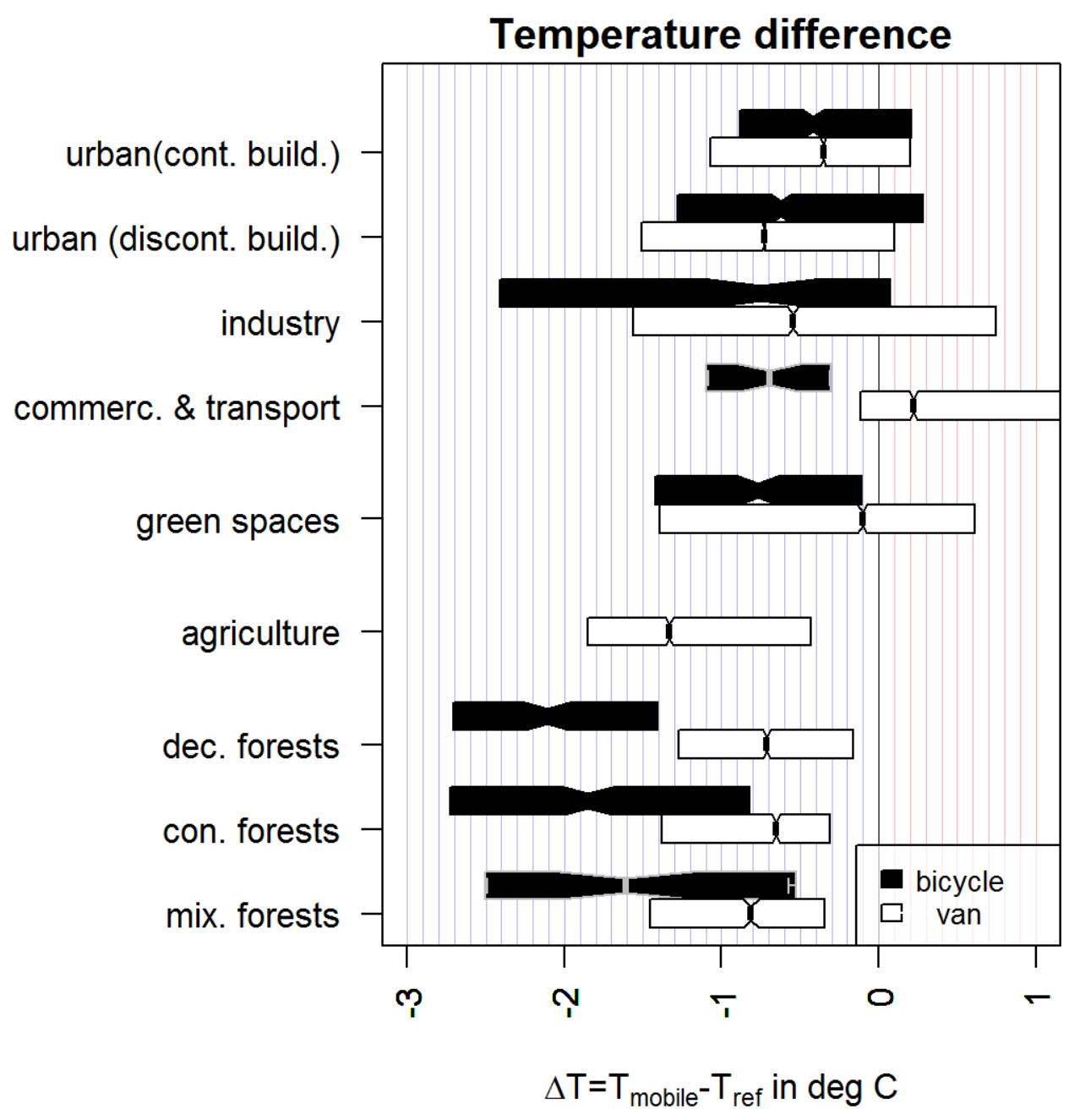

Figure S5.1 Boxplot of temperature differences for different land use types and different observation platforms compared to the measurements in Berlin-Neukölln. Displayed is the range between the $25^{\text {th }}$ and the $75^{\text {th }}$ percentile with a notch from $45^{\text {th }}$ to $55^{\text {th }}$ percentile. Grey borderlines of bars represent categories with insufficient data values, missing bars no data by the corresponding method. 
Table S5.2. Changes in air temperature between mobile measurements and the reference site in Berlin-Neukölln (BLUME). Values are provided as $\Delta \mathrm{T}$ (“bicycle")/ $\Delta \mathrm{T}$ ("van") both in degrees $\mathrm{C}$. The value is negative if the mobile measurement recorded cooler values.

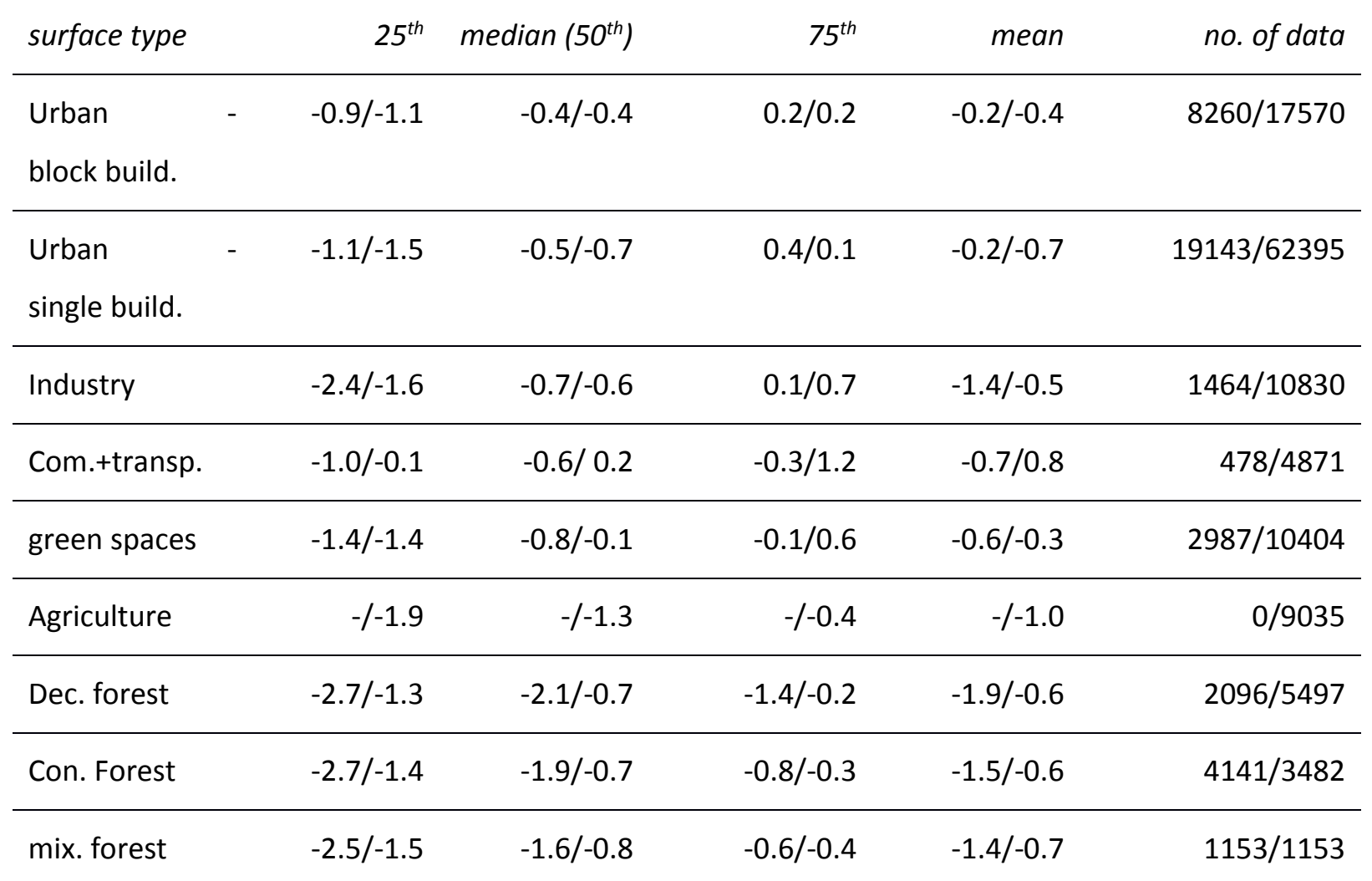


S6. Comparison of different measurement methods with reference ones in Neukölln

\section{S6.1 Ozone}
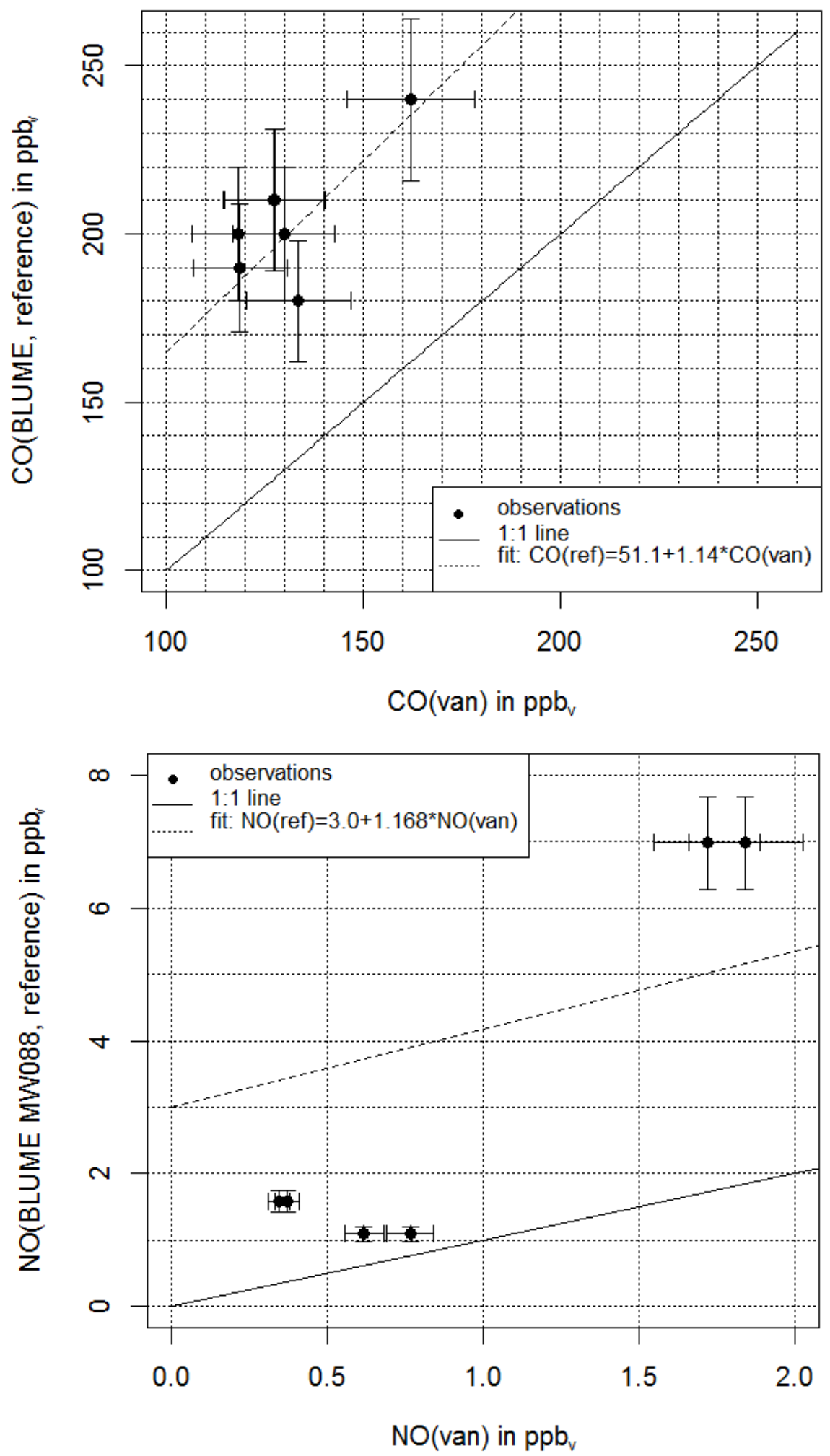

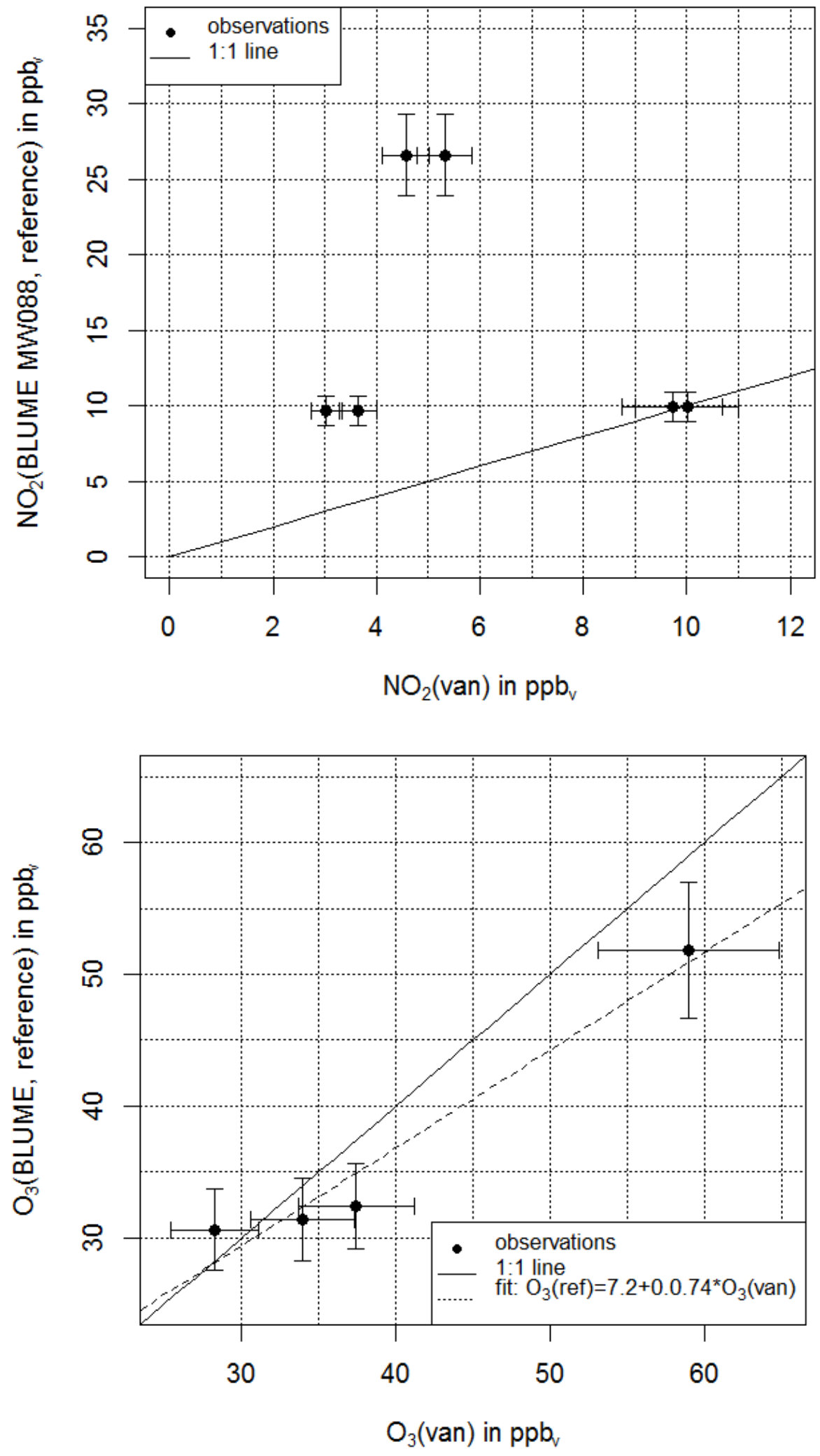

Fig. 6.1.1. Comparison for $\mathrm{CO}, \mathrm{NO}, \mathrm{NO} 2$ and $\mathrm{O} 3$ at the stationary reference site. Note, while long term stable compounds match best, small scale and temporal variable gases such as NO do not. Any of the mobile instruments was calibrated a priori to the campaign and BLUME instruments earlier the year. 


\section{S6.2. Particulate mass comparisons for different measurement techniques}

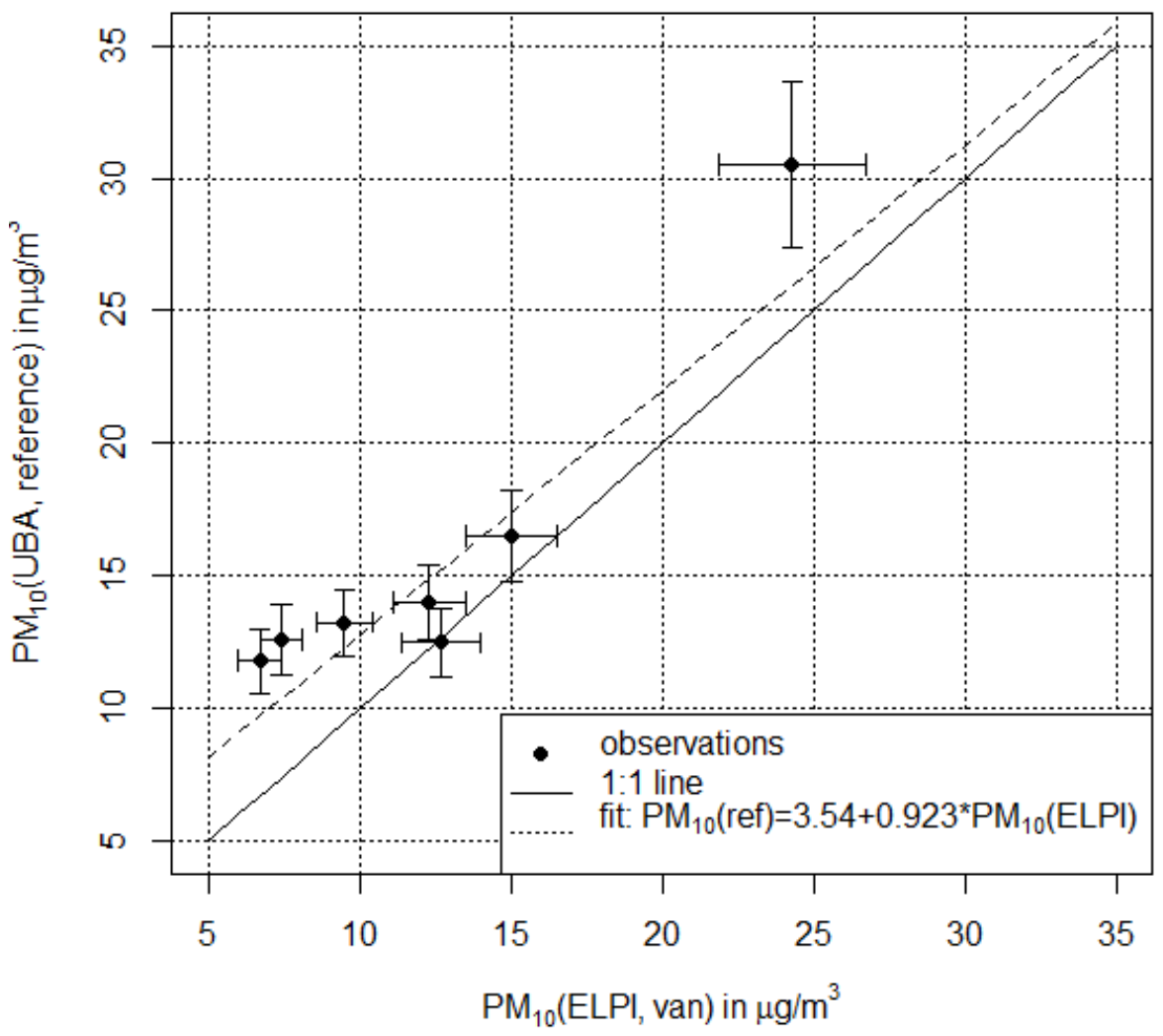

Comparison of mean values for bicycle and BLUME measurements

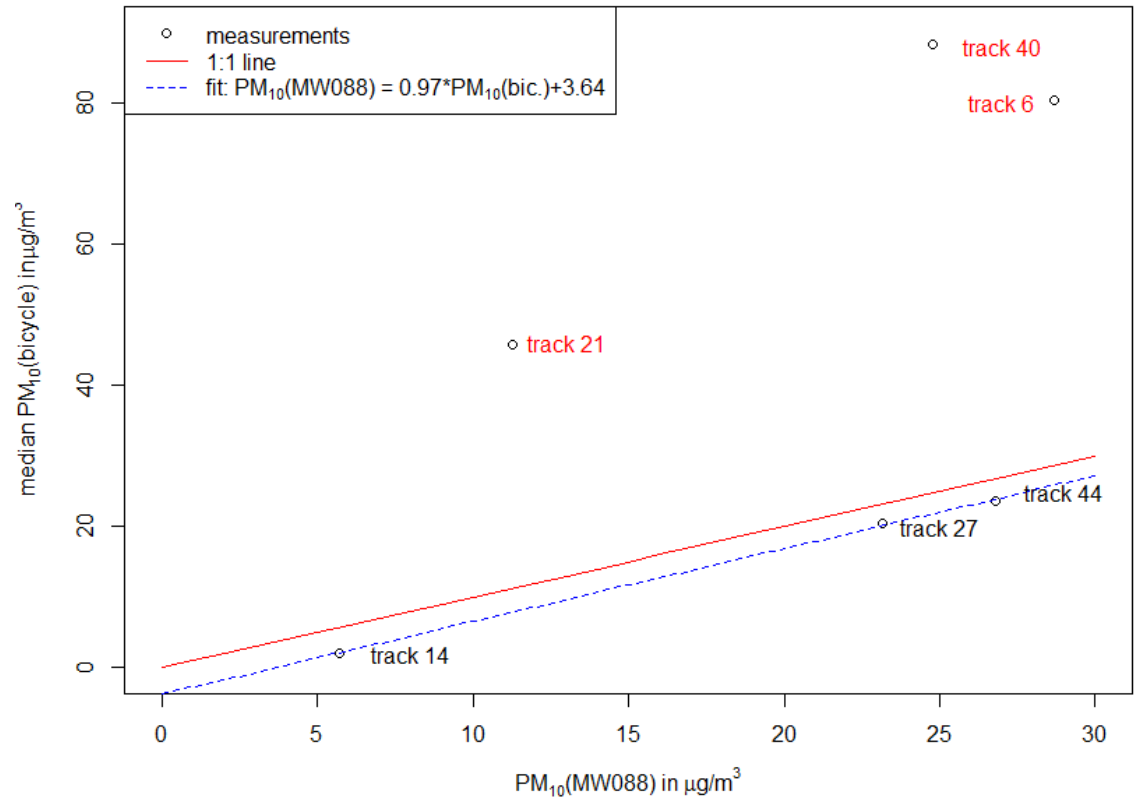

Fig. 6.2.1. Comparison of mobile observed PM10 values (top: van, bottom: bicycle based) with 30 min averages at the reference site. Three short stops (no. 6, 21 and 40) of the cyclists seem to be notably affected by short term local pollution of very large particles. 
Comparison of mean values for bicycle and BLUME measurements

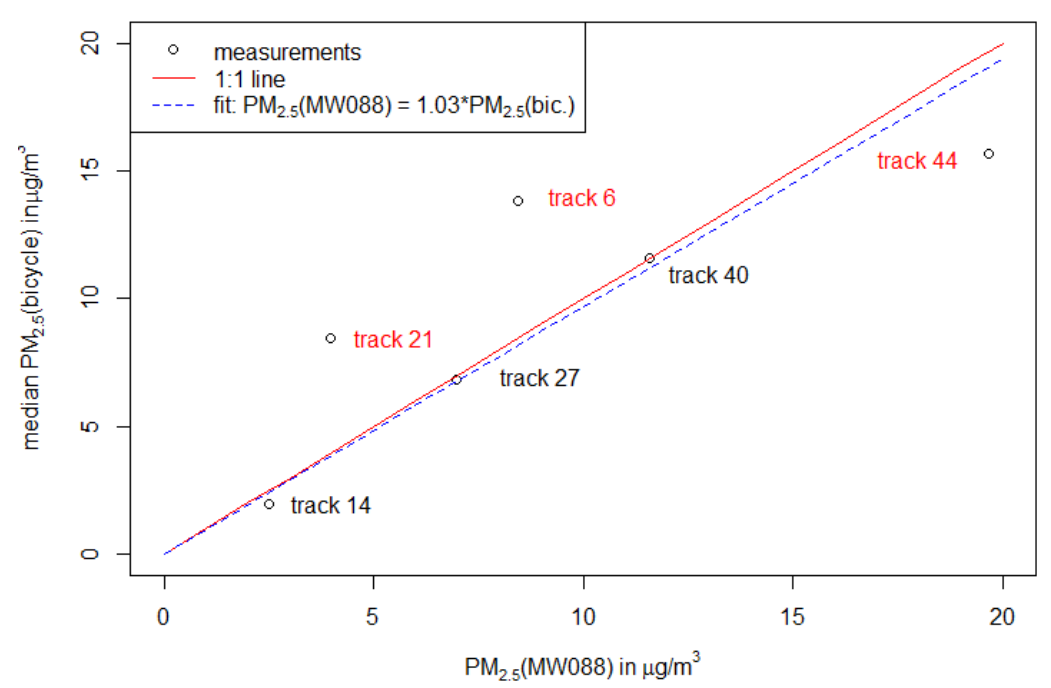

Comparison of mean values for bicycle and BLUME measurements

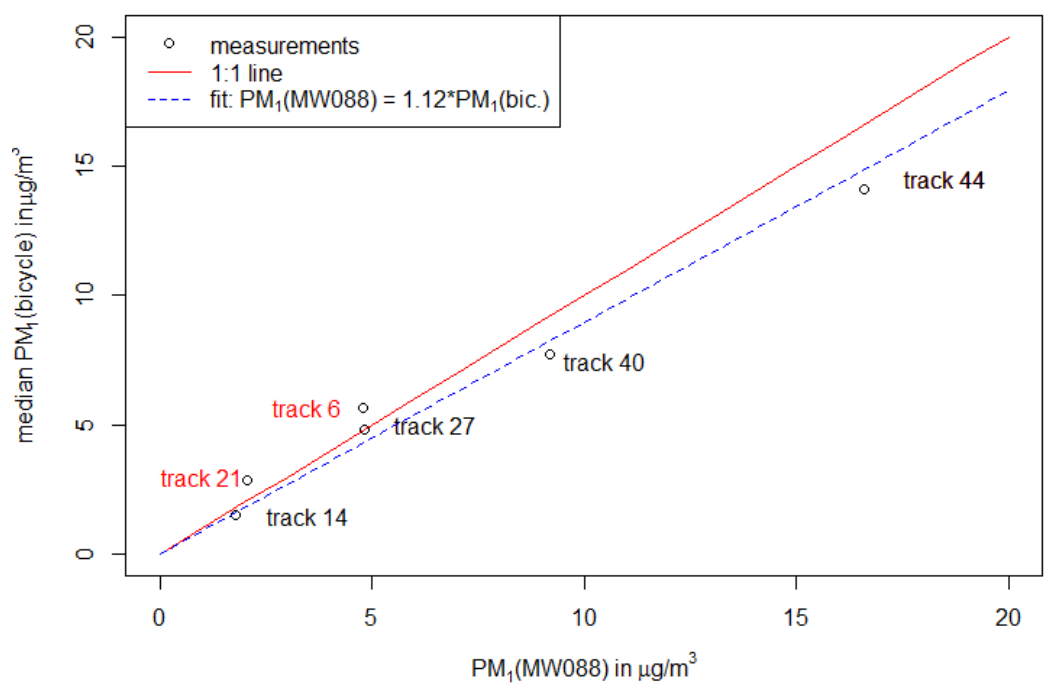

Fig. 6.2.2. $\quad \mathrm{PM}_{2.5}$ and $\mathrm{PM}_{1}$ comparison as for $\mathrm{PM}_{10}$ in above Figure 6.1 .1 but for bicycles only. Other values than $\mathrm{PM}_{10}$ for van measurements were not available. In here affected bicycle track measurements hardly apparent. Effect predominantly on largest sizes. 
Comparison of mean values for bicycle and BLUME measurements

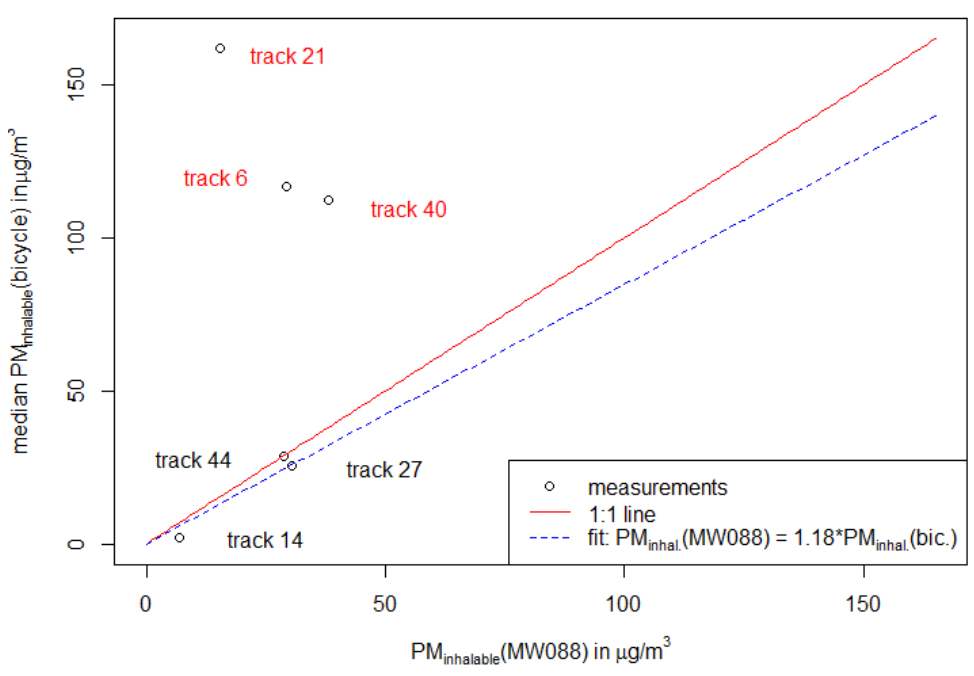

Comparison of mean values for bicycle and BLUME measurements

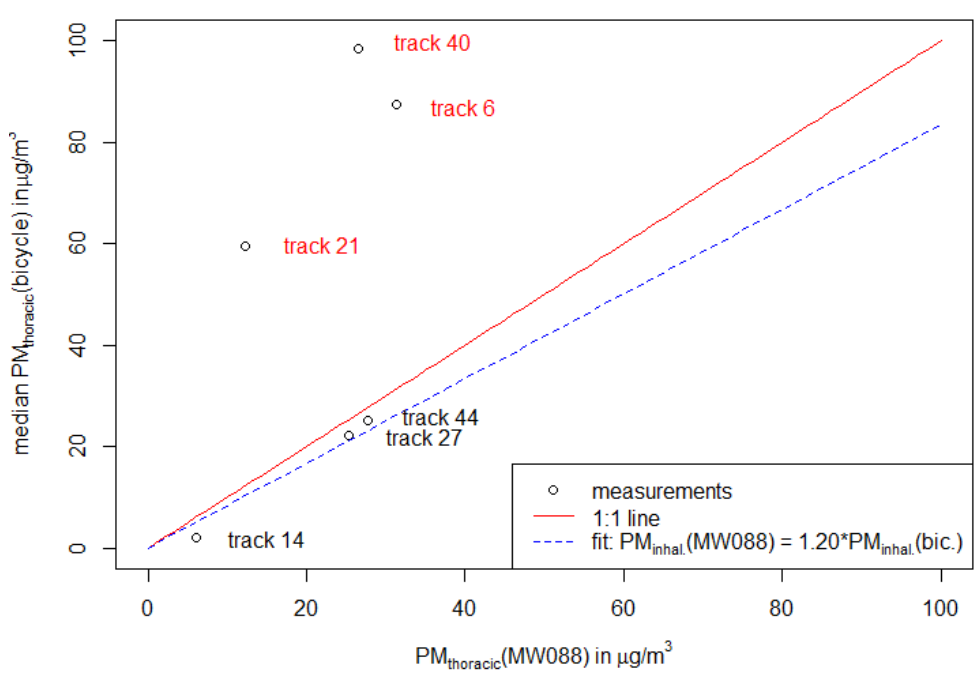

Comparison of mean values for bicycle and BLUME measurements

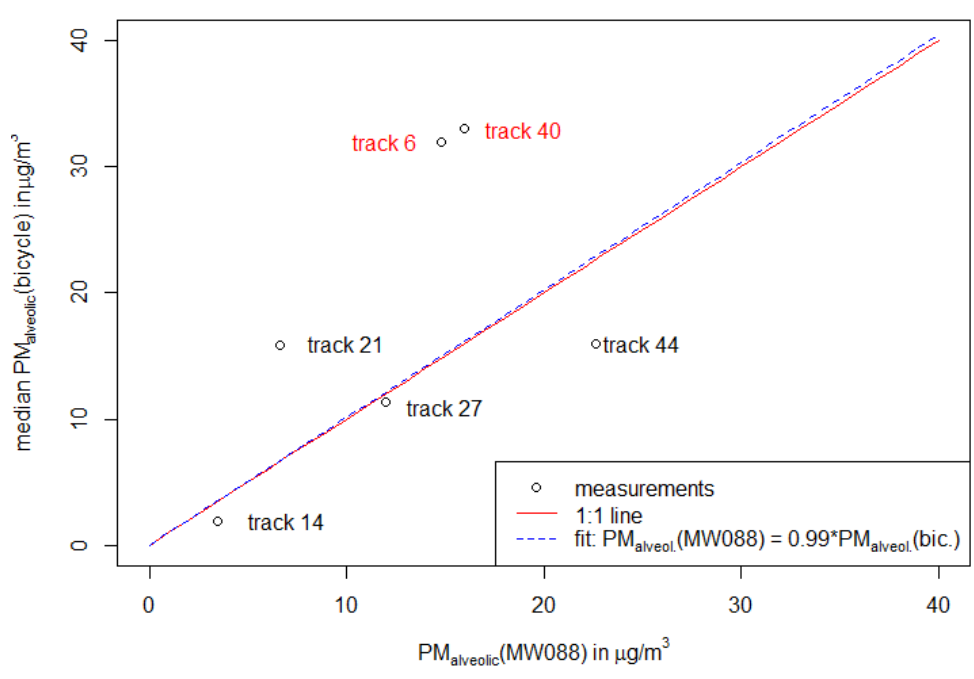

Fig. 6.2.3 Health related parameterized $P M$ value comparison as for $\mathrm{PM}_{10}$ in above Figure 6.2.1 but for bicycles only. Other values than $\mathrm{PM}_{10}$ for van measurements were not available. 


\section{S6.3 Particle number concentration (PNC)}
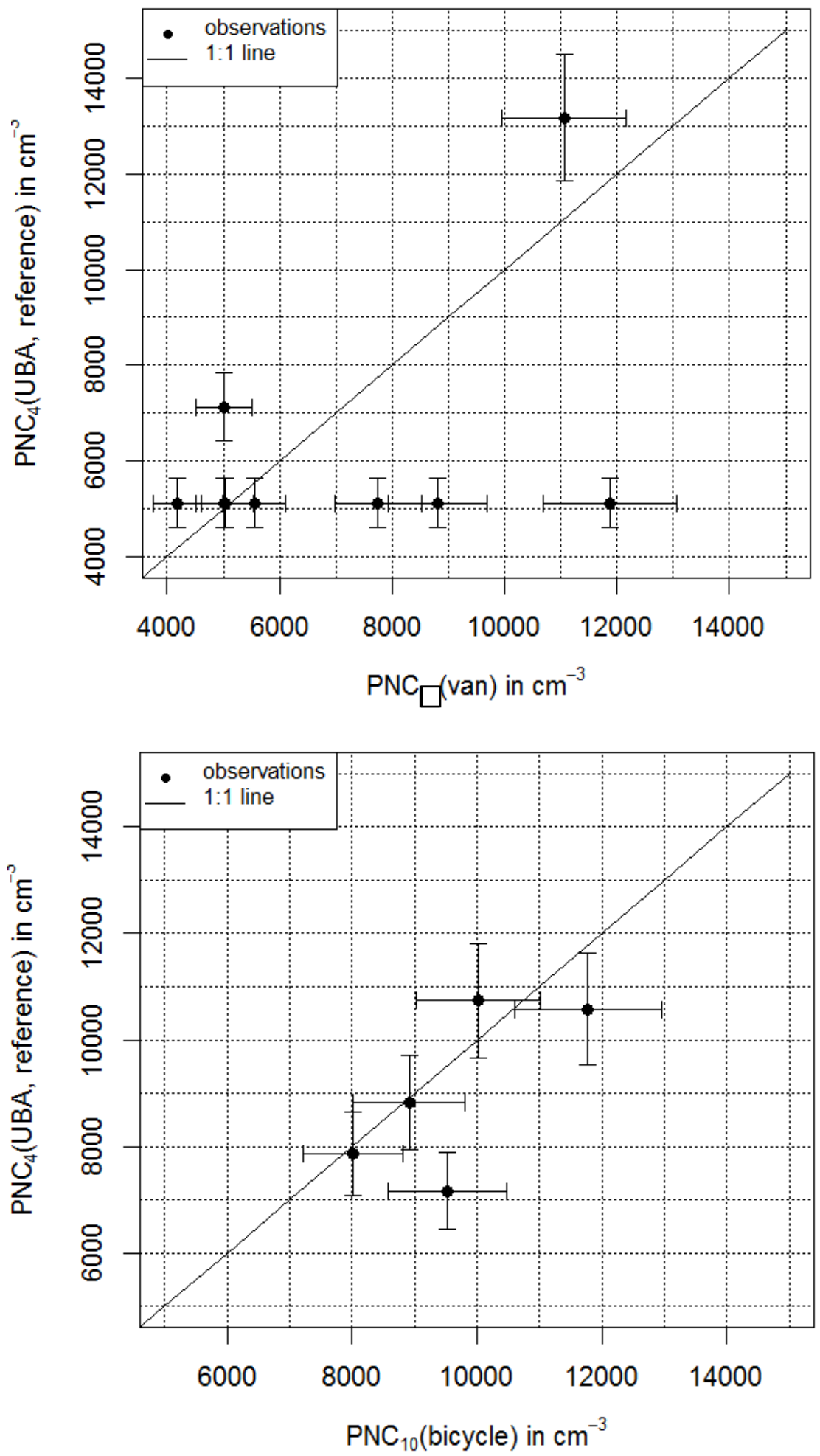

Fig. 6.3. Particle number comparison with reference site measurements, top: van, bottom and bicycle based measurements with different lower cut-off diameters, i.e. van: $3 \mathrm{~nm}$ and bicycle: $10 \mathrm{~nm}$. 